ARTICLE

\title{
Data-informed discovery of hydrolytic nanozymes
}

\author{
Sirong $\mathrm{Li}^{1}$, Zijun Zhou${ }^{1}$, Zuoxiu Tie ${ }^{1}$, Bing Wang${ }^{2}$, Meng Ye ${ }^{3}$, Lei Du ${ }^{4}$, Ran Cui ${ }^{4}{ }^{4}$, Wei Liu ${ }^{3}$, Cuihong Wan², \\ Quanyi Liu ${ }^{5,6}$, Sheng Zhao ${ }^{1}$, Quan Wang ${ }^{1}$, Yihong Zhang ${ }^{1}$, Shuo Zhang ${ }^{1,7}$, Huigang Zhang (1) ${ }^{1,7}$, Yan Du $^{5,6}$ \& \\ Hui Wei (10) 1,8,9凶
}

Nanozyme is a collection of nanomaterials with enzyme-like activity but higher environmental tolerance and long-term stability than their natural counterparts. Improving the catalytic activity and expanding the category of nanozymes are prerequisites to complement or even supersede enzymes. However, the development of hydrolytic nanozymes is still challenged by diverse hydrolytic substrates and following complicated mechanisms. Here, two strategies are informed by data to screen and predict catalytic active sites of MOF (metal-organic framework) based hydrolytic nanozymes: (1) to increase the intrinsic activity by finely tuned Lewis acidity of the metal clusters; (2) to improve the density of active sites by shortening the length of ligands. Finally, as-obtained Ce-FMA-MOF-based hydrolytic nanozyme is capable of cleaving phosphate bonds, amide bonds, glycosidic bonds, and even their mixture, biofilms. This work provides a rational methodology to design hydrolytic nanozyme, enriches the diversity of nanozymes, and potentially sheds light on future evolution of enzyme engineering.

\footnotetext{
${ }^{1}$ College of Engineering and Applied Sciences, Nanjing National Laboratory of Microstructures, Jiangsu Key Laboratory of Artificial Functional Materials, Nanjing University, 210023 Nanjing, Jiangsu, China. ${ }^{2}$ Hubei Key Laboratory of Genetic Regulation and Integrative Biology, School of Life Sciences, Central China Normal University, 430079 Wuhan, Hubei, China. ${ }^{3}$ Jiangsu Key Laboratory of Druggability of Biopharmaceuticals, State Key Laboratory of Natural Medicines, School of Life Science and Technology, China Pharmaceutical University, 211198 Nanjing, Jiangsu, China. ${ }^{4}$ College of Chemistry and Molecular Sciences, Wuhan University, 430072 Wuhan, Hubei, China. ${ }^{5}$ State Key Laboratory of Electroanalytical Chemistry, Changchun Institute of Applied Chemistry, Chinese Academy of Sciences, 130022 Changchun, Jilin, China. ${ }^{6}$ University of Science and Technology of China, Hefei, 230026 Anhui, Hefei, China.

${ }^{7}$ Collaborative Innovation Center of Advanced Microstructures and Institute of Materials Engineering Nanjing University, 210093 Nanjing, Jiangsu, China.

${ }^{8}$ State Key Laboratory of Analytical Chemistry for Life Science, School of Chemistry and Chemical Engineering, Nanjing University, 210023 Nanjing, Jiangsu,

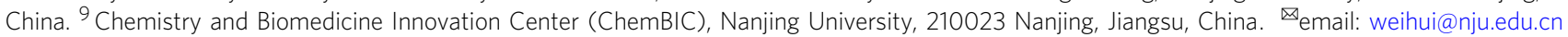


xploring enzyme mimics in artificially fabricated systems is - a promising strategy to overcome the instability and high cost of enzymes ${ }^{1}$. It can also enable us to better understand the living world ${ }^{2}$. However, the development of artificial enzymes is challenged by limited knowledge on miscellaneous mechanisms and finite chemical methodology to mimic. Over the last two decades, the emergence of nanotechnology has expanded artificial enzymes into nanomaterials, which are now collectively termed as nanozymes $^{3-7}$. Nanozymes integrate multivalent catalytic sites while retaining the multifunctional repertoires of nanomaterials, such as the magnetic property of $\mathrm{Fe}_{3} \mathrm{O}_{4}$. Thereby diverse feats in bioanalysis ${ }^{4,8,9}$, medical imaging ${ }^{10}$, therapeutics ${ }^{11}$ and tissue engineering ${ }^{12}$ have been achieved, enriching biomimetic nanozymes to a larger context.

In our recent review on nanozymes ${ }^{3}$, we summarised an exponential growth in the number of publications on nanozymes, demonstrating the fast expansion of this field. However, the breadth of enzymatic reactions that has been explored is, to date, rather limited. Specifically, a detailed analysis of these papers showed that only a small fraction (7.1\%) focused on hydrolytic enzyme mimics, while the majority (92.9\%) focused on redox enzyme mimics (Fig. 1a). To fully exploit nanozymes, it is demanded to widen the category that nanozymes can mimic and study them in-depth to compensate this 13 -fold difference in the number of studies on hydrolase and redox enzyme mimics.
Biologically, hydrolases are involved in 13 different biotransformations. Among them, esters (esterases), phosphoesters (phosphoesterases), amides (amidases and proteases), and carbohydrates (glycosidases) are the most common ones, see in Supplementary Table 1 . The broad substrate scope of hydrolases enables them to function in nerve impulse transmission (e.g., acetylcholinesterase), blood sugar balance (e.g., glycogen phosphorylase), dissimilation (e.g., digestive enzyme) and energy transfer (e.g., ATP hydrolase). Additionally, organophosphatelike nanozymes can degrade organophosphorus pesticides/chemical warfare agents (such as soman, sarin, and tabun) by breaking the $\mathrm{P}-\mathrm{X}$ bond $(\mathrm{X}=\mathrm{O}, \mathrm{F}, \mathrm{CN} \text {, etc. })^{13}$, hopeful to be embedded into protective masks. Despite the encouraging achievements, to date, only a few such nanozymes have been developed, which can be attributed, at least in part to that the current design strategies are heavily relying on integrating natural active site moieties (such as $\mathrm{Zn}^{2+}$-coordinated complexes) within/onto nanomaterials ${ }^{5,14-19}$. The specificity and regulation of natural active sites restrict the further evolution of these nanozymes in abiotic environment or specific microenvironment, which may lead to non-optimal reactivity and thus an unsuitable replication template for new-to-nature catalysis ${ }^{20-22}$. Moreover, the diversity of substrates and relatively limited knowledge on the catalytic mechanisms of hydrolases give additional obstacles towards the design of new hydrolytic nanozymes. To overcome (a)

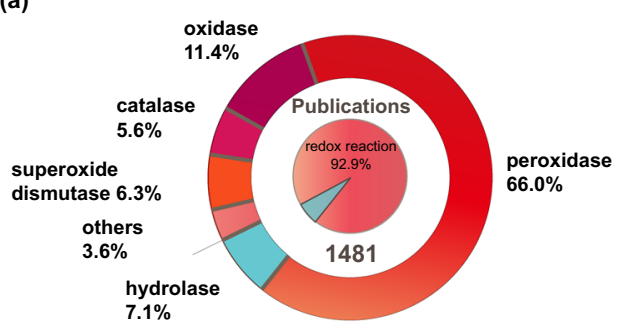

(d) (b)

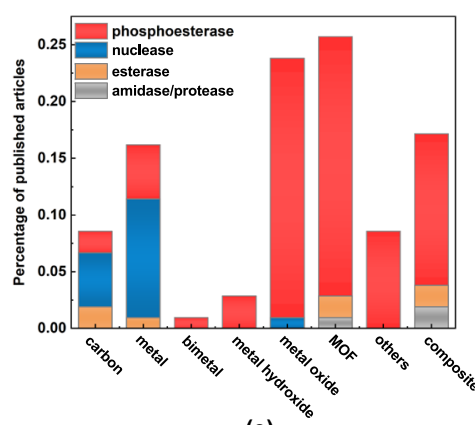

(c)

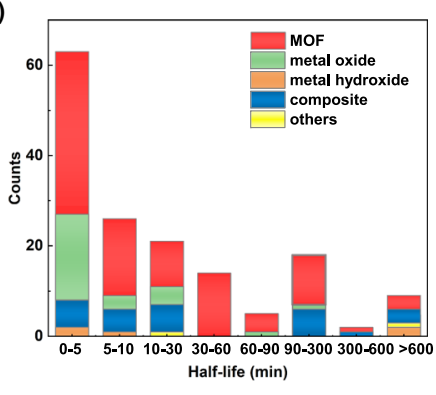

(e)
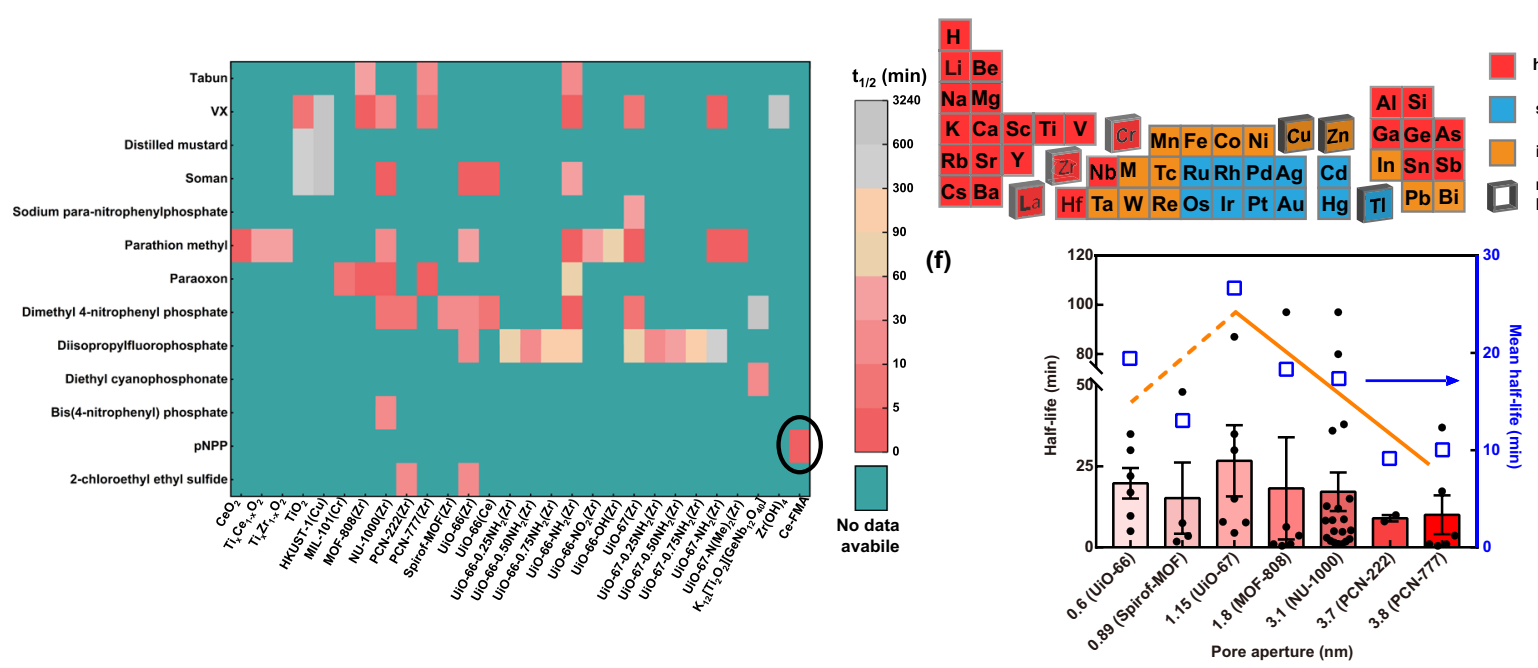

Fig. 1 The overall statistical analysis of hydrolytic nanozymes. a Proportions of different types of nanozymes. Raw data were adapted from Wu et al. ${ }^{3}$. b Stacked histogram of the publication frequency distribution of studies on different types of material related to each type of hydrolase. c Stacked histogram of the number distribution of each material based on its half-life of phosphatase-like activity. $\mathbf{d}$ Heat map of the half-lives of various phosphatase-like nanozymes and their corresponding substrates. Each half-life is presented as the mean unless there is only one sample. The circled one represents the data-informed Ce-FMA in this work. e Periodic table of metal elements mapped by hard-soft-acid-base theory. The red indicates hard Lewis acid, the blue indicates soft Lewis acid and the yellow indicates the intermediate Lewis acid. Titled squares represent metal compositions that have been reported with hydrolase-like activity. $\mathbf{f}$ Half-lives of reported $\mathrm{Zr}$-based MOFs versus their pore aperture; the mean values are shown as blue empty squares. The number associated with each MOF on the $\mathrm{x}$-axis represents the pore/window aperture ${ }^{14,40,47-51}$. Each half-life is presented as the mean \pm standard deviation. 
these challenges and expand nanozyme functionality beyond nature's repertoire, herein, we identified two key factors to design MOF (metal-organic framework) based hydrolytic nanozymes through a data-informed analysis of published hydrolase-like nanozymes based on our recent comprehensive review ${ }^{3}$.

Data-informed analysis is an approach that balances between expertise and understanding of information, thus drawing key information and further deriving insights from structured and unstructured data ${ }^{23}$. In this work, 105 research papers describing hydrolytic nanozymes are screened from 1481 research papers we reviewed (the list of papers we covered is supplemented as Supplementary Data 1). The breadth and the efficiency heat map of hydrolytic nanozymes (Fig. 1d) indicated MOF as a good scaffold to incorporate active sites. Further, two factors were informed through data of which the metal ions were suggested as hard Lewis acid $\left(i . e ., \mathrm{Ce}^{4+}\right.$ ) for higher affinity with substrates while the ligand was deduced as fumaric acid in that a shorter ligand could increase the density of the active sites ${ }^{24}$. The optimised hydrolytic MOF nanozyme was prepared experimentally and shown to possess excellent phosphatase-, protease- and glycosidase-like activities among current nanozymes. Moreover, the optimised hydrolytic nanozyme even successfully degraded biofilms under mild conditions. This work provides a general methodology to design hydrolytic nanozymes. And the abiotic active sites we derived may conversely shed a light on enzyme engineering in the future.

\section{Results}

Data-informed strategy to identify hydrolase-like material candidates. The course to develop a potent hydrolytic nanozyme is (1) selecting a suitable scaffold to embed/design highly active sites; (2) identifying a highly reactive site to activate hydrolytic substrates; and (3) performing experiments to fabricate and confirm the designed materials. We envisioned the suitable scaffold to embed active sites for nanozymes can be deduced from data classified by varying material category. Therefore, we first plotted the publication frequency distribution of studies on different types of material related to each type of hydrolase in Fig. 1b. Four types of hydrolase, namely, phosphoesterase, nuclease, esterase, and amidase/protease (there were no glycosidase mimics when this work was initiated in mid-2018) were sorted which were mimicked by carbon, metal, bimetal, metal hydroxide, metal oxide, MOF, composite and others. Notably, reports on MOFs, which are crystalline materials consisting of metal clusters coordinated by organic ligands ${ }^{25-27}$, and metal oxides were the most numerous, indicating these two as scaffold candidates to mimic hydrolases. On the other hand, half-life $\left(\mathrm{t}_{1 / 2}\right.$, the time needed to achieve $50 \%$ conversion) which was presented as the most popular kinetic parameter among collected data, is grouped in Fig. 1c to determine which scaffold is more likely to achieve faster catalytic speed. MOFs quickly draw a specific focus because of their much higher representation in the literature than other materials with a sub-10 min half-life. Consequently, we inferred that MOFs are optimal candidates to mimic hydrolases.

Having confirmed MOFs as a potent scaffold, our next goal was to deduce highly active sites of MOFs. A kinetic heat map that displayed the half-lives of various phosphorylated substrates treated with nanozymes was shown in Fig. 1d. Intriguingly, though dozens of materials were reported to mimic hydrolase, the metal components were within six elements, namely zirconium (IV), cerium (IV), chromium (III), copper (II), zinc (II) and titanium (IV), see the tilted box in Fig. 1e. Moreover, nanozymes consisted of metal ions with hard Lewis acidy (see the Lewis acidy summarised in Supplementary Table 2) tend to have faster half-lives, such as zirconium (IV), the major (17/20) component of the hydrolytic MOFs, manifesting hard Lewis acid as effective active sites.
Mechanistically, a hard Lewis acid, such as a high-valence metal ion, can easily activate a carbonyl or phosphoryl group by accepting an electron lone pair from the oxygen and drawing electron density away from the double bond, leading to a greater positive charge on -and thus increasing the electrophilicity and reactivity of-the central carbon or phosphorus (see in Supplementary Figure 1) ${ }^{28,29}$. Thus, Lewis acid metal ions are preferred active sites in a MOF scaffold for mimicking hydrolases, based on both an analysis of previously published data and hard-soft-acid-base theory.

Another impact factor in MOF scaffolds is the ligand which controls the connectivity, topology and spacing/density of metal clusters and moreover affects the final pore/window aperture of MOFs. Since different connectivity between the same metal ion and ligand can yield MOFs with different topology structures and pores/windows, we classified the MOFs in the abovementioned papers by their pore/window aperture instead of ligand and plotted their kinetic data (half-lives) in Fig. 1f. For MOFs with pore widths above approximately $1 \mathrm{~nm}$, larger pores lead to faster reaction rates (i.e., shorter half-lives) due to easier substrate diffusion into the catalytic interior of the MOF (solid yellow trend line, Fig. $1 \mathrm{f})^{28}$. Below pore widths of approximately $1 \mathrm{~nm}$, however, this trend is reversed. We attributed this reversed trend to the increased density of active site as shorter ligand means greater vicinity of metal clusters ${ }^{24}$. Of the MOFs in which this trend is observed, UiO-66 and UiO-67 are of particular interest, as these two isostructural MOFs differ only in the length of their structural ligands (shorter ligand benzene-1,4-dicarboxylic acid (BDC) in UiO-66 and longer ligand biphenyl-4,4'-dicarboxylate (BPDC) in UiO-67; Supplementary Fig. 2). In this regard, we selected fumaric acid (FMA, Supplementary Figure 3) as a candidate ligand because its length is shorter than BDC but able to form/construct a homologue of UiO-66. Modulators have been demonstrated to affect the yield ${ }^{30}$, degree of crystallinity ${ }^{30}$, morphology/size ${ }^{17}$ and presence of defects ${ }^{29}$ of the synthesised $\mathrm{MOFs}^{30}$, ultimately resulting in differences in the catalytic performance. Therefore, we also screened modulators. Three modulators, acetic acid (AA), formic acid (FA) and trifluoroacetic acid (TFA) (see their structures in Supplementary Fig. 4), were outlined for the similar carboxylic acid structures to that of FMA. Of note, though FMA contained UiO-66 like MOFs have been synthesised ${ }^{31,32}$, there currently are no available data showing that an FMA contained MOF possesses hydrolase-like activity.

Based on the above analyses, we selected high-valence, strong Lewis acid ions $\left(\mathrm{Zr}^{4+}, \mathrm{Ce}^{4+}\right.$, and $\mathrm{Hf}^{4+}$, the three tetravalent metal ions with the strong Lewis acidity in Supplementary Table 2), FMA and modulators to construct a homologue MOF of UiO-66. Then, a total of 15 kinds of substrates (summarised in Supplementary Table 3) towards four different hydrolases were involved to confirm its activity.

Optimisation of the metal ions and ligands. The protocols to fabricating Zr-FMA, Hf-FMA and Ce-FMA was described in Methods section. As shown in Supplementary Fig. 5a, crystalline Ce-FMA could be collected within $10 \mathrm{~min}$ at room temperature. Therefore, Ce-FMAs modulated by different modulators with varied ratios were collected after 10 min stirring (Supplementary Fig. 6), yielding MOFs with different size (Supplementary Fig. 7) as well as distinct Brunner-Emmet-Teller (BET) surface area (Supplementary Fig. 8 and Supplementary Table 6). In addition to Zr-FMA, Hf-FMA and Ce-FMA, we fabricated Zr-BDC, Hf$\mathrm{BDC}$ and Ce-BDC to investigate which metal ions dominate the hydrolytic activity and whether increasing the density of active sites by shortening the length of ligand can increase the phosphatase activity towards p-nitrophenyl phosphate (pNPP) and bis-p-nitrophenyl phosphate (BNPP). 
(a)

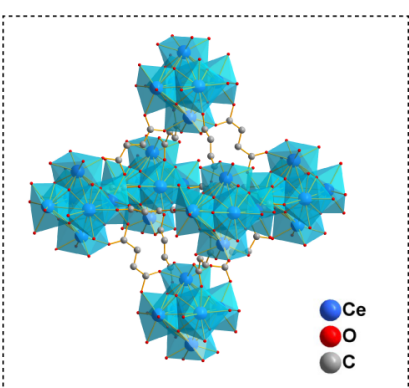

(d)

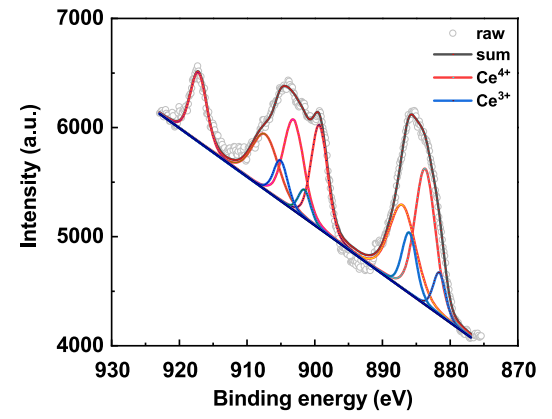

(b)

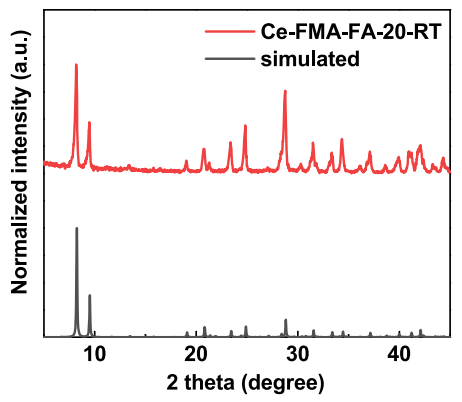

(e)

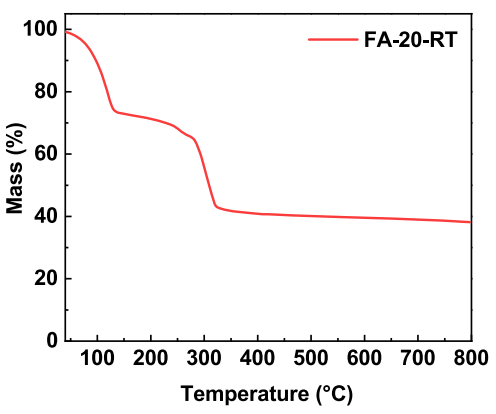

(c)

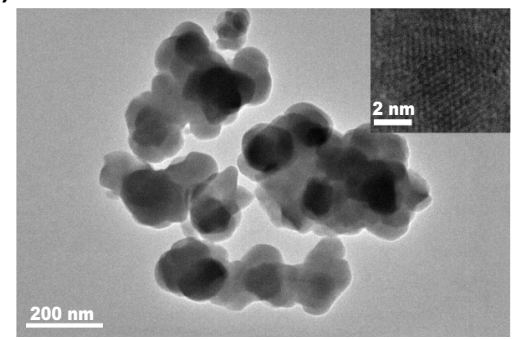

(f)

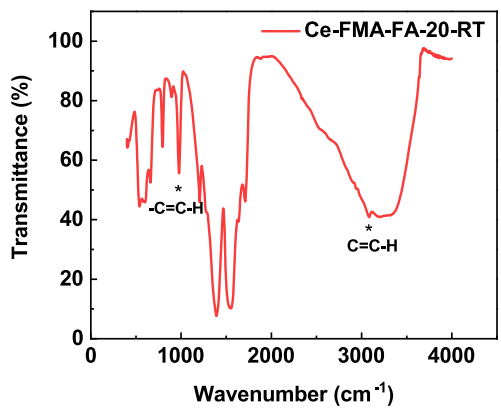

Fig. 2 Characterisation of Ce-FMA modulated by FA with an FA-to-FMA molar ratio of 20. a Structure of Ce-FMA. Ce blue, O red, C grey; hydrogen atoms are omitted for clarity. $\mathbf{b}$ XRD pattern of Ce-FMA-FA-20-RT (the black lines at the bottom are simulated patterns from Lammert et al. ${ }^{31}$, with CCDC number 1036903). c TEM images of Ce-FMA-FA-20-RT at different magnifications. d XPS spectrum of the Ce 3d signals of the Ce-FMA-FA-20-RT. The red curves correspond to $\mathrm{Ce}^{4+}$ which are deconvoluted into three Voigt doublets and the blue curves correspond to $\mathrm{Ce}^{3+}$ which are deconvoluted into two Voigt doublets. The black curve is summed by every deconvoluted peak. e TG curve of Ce-FMA-FA-2O-RT analysed under air flow. $\mathbf{f}$ FTIR spectrum of CeFMA-FA-20-RT.

Having confirmed the identical phase structure of six MOFs by $\mathrm{XRD}$, as shown in Supplementary Fig. 9a, we then tested phosphatase-like activity towards pNPP. Neither Hf-BDC nor HfFMA showed prominent phosphatase-like activity, as shown in Supplementary Fig. 9b, c, and these samples therefore served as null examples of Hf-based hydrolytic nanozymes. We then compared Zr- and Ce-based MOFs linked by FMA and BDC. Consistently, the Ce-based systems demonstrated greater catalytic activity than the Zr-based systems (Supplementary Fig. 9d, e), and nonactivated Ce-FMA demonstrated higher activity than activated Ce-BDC (Supplementary Fig. 9f), even though Ce-FMA and Ce-BDC showed similar size in Supplementary Fig. 10. Of note, the BET surface area of Ce-FMA was $\sim 4$ times smaller than that of activated Ce-BDC $\left(120.44 \mathrm{~m}^{2} / \mathrm{g}\right.$ versus $517.00 \mathrm{~m}^{2} / \mathrm{g}$, respectively; Supplementary Table 6). This difference between the six MOFs, for one thing, demonstrates our hypothesis that a shorter ligand is beneficial to yield higher density to the active sites; for another, indicates that the increased Lewis acidity of $\mathrm{Ce}$ makes the $4 f$ orbital well suited to hybridise with the P-O bond and thus better stabilises the pentavalent phosphate intermediate for nucleophilic attack. Both factors collectively make the CeFMA as particularly well-suited for a hydrolase-like catalys ${ }^{33}$.

Characterisation of the optimal Ce-FMA-FA-20-RT. An overall comparison of the various synthetic conditions for Ce-FMA was conducted via phosphatase-like activity assays with the substrates pNPP and BNPP (Supplementary Fig. 11). Both of the mass activity and surface area-normalised activity towards pNPP and BNPP were taken into consideration to optimise the synthetic conditions of Ce-FMA (Supplementary Fig. 11b-e) since it is convenient to use mass activity to evaluate cost, but more consistent with the active sites by surface area-normalised activity. Finally, an FA-to-FMA molar ratio of 20 was determined to be the best synthetic condition according to the phosphatase-like activity, and this optimised structure was referred to as Ce-FMAFA-20-RT. As illustrated in Fig. 2a, the $\left[\mathrm{Ce}_{6} \mathrm{O}_{4}(\mathrm{OH})_{4}\right]^{12+}$ clusters are arranged as cubic close packing and linked by $12 \mathrm{FMA}^{2-}$, yielding the formula as $\left[\mathrm{Ce}_{6} \mathrm{O}_{4}(\mathrm{OH})_{4}(\mathrm{FMA})_{6}\right]$ in Ce-FMA. The XRD patterns in Fig. 2b confirmed consistent crystal structure with simulated results ${ }^{31}$. TEM images revealed that this modulated MOF was $\sim 200 \mathrm{~nm}$ in diameter with slight aggregation (Fig. 2c). X-ray photoemission spectroscopy (XPS) deconvolution of the peaks of Ce $3 d$ in Fig. 2d indicates a proportion of $82.7 \%$ $\mathrm{Ce}^{4+}$ and $17.3 \%$ of $\mathrm{Ce}^{3+}$, making the final valence of 3.8 . A typical three-stage thermal behaviour determined by thermogravimetric analysis was also exhibited (Fig. 2e), suggesting a similar thermal behaviour with Ce-BDC ${ }^{31}$. Fourier transform infrared spectroscopy (FTIR) in Fig. 2f also demonstrates the remained trans structured $\mathrm{C}=\mathrm{C}$ in MOFs derived from FMA.

Catalytic performance. A total of 15 different substrates (listed in Supplementary Table 3) towards four main hydrolases were applied to evaluate the catalytic performance of Ce-FMA-FA-20-RT. Since the phosphoester bond is the most active bond among the above hydrolytic bonds, we studied phosphatase-like activity first and used the activity data to verify the optimal synthetic conditions (confirming the modulator and the dosage) of Ce-FMA. Then we explored Ce-FMA to hydrolyse planar amide bond in bovine serum albumin (BSA) which is more hierarchal in structures and difficult to cleave. With such success, we next investigated glycoside bonds cleavage effect in chitosan. In summary, except for carbonate esterase, Ce-FMA is able to mimic the other three types of hydrolase.

Phosphatase-like activity. Despite the variation in all the phosphorylase substrates previously reported (summarised in Fig. 1d), the catalytic mechanism was consistent with Lewis acid-activated 
(a)

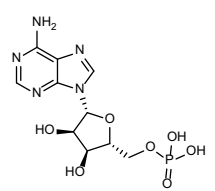

adenosine monophosphate

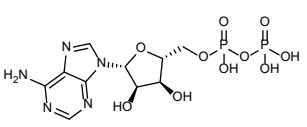

adenosine diphosphate

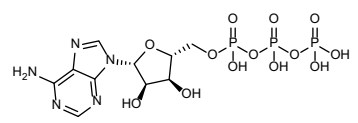

adenosine triphosphate

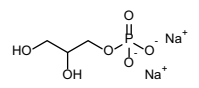

sodium $\beta$-glycerophosphate (b)

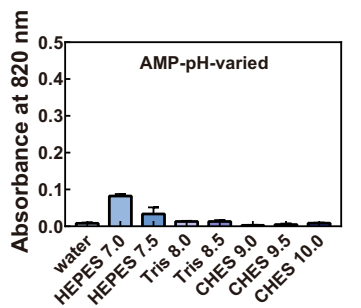

(c)

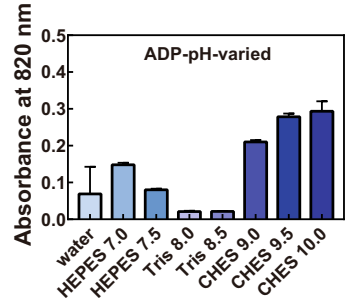

(d)

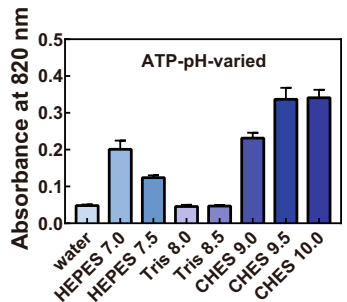

(e)

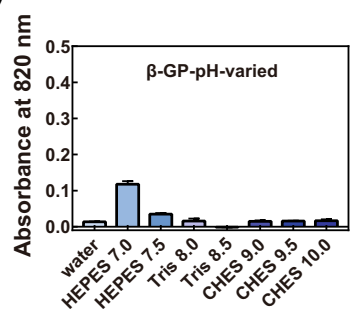

Fig. 3 Phosphatase-like activity of Ce-FMA towards AMP, ADP, ATP and $\boldsymbol{\beta}$-GP. a Chemical structures of AMP, ADP, ATP and $\beta$-GP. pH-dependent cleaving activity of Ce-FMA-FA-20-RT towards AMP (b), ADP (c), ATP (d) and $\beta$-GP (e). Experiments were carried out at $37^{\circ} \mathrm{C}$ with $1 \mathrm{mM}$ substrate and $0.1 \mathrm{mg} / \mathrm{mL}$ Ce-FMA-FA-20-RT for $12 \mathrm{~h}$. Data are processed by removing the blank groups (without catalyst), and presented as the mean \pm standard error, $n=4$. The conversion rate of $12 \mathrm{~h}$ towards AMP, ADP ATP and $\beta$-GP under the optimised $\mathrm{pH}$ is summarised in Supplementary Table 7.

cleavage of the $\mathrm{P}-\mathrm{X}$ bond $(\mathrm{X}=\mathrm{O}, \mathrm{F}, \mathrm{CN}$, etc.). For phosphorylated substrates, as illustrated in Supplementary Fig. 1, the reaction starts with the nucleophilic addition of an undercoordinated $\mathrm{M}-\mathrm{OH}$ (M refers to metal) after the substrate was activated by metal cluster in MOF, forming a pentacoordinated phosphorus intermediate. And then, the intermediate decomposed via the elimination of alcohol ${ }^{34}$. Since most of the organophosphorus are neurotoxic, for analytical efficiency and safety, we selected pNPP and BNPP as phosphatase substrates instead of the other organophosphorus compounds. As shown in Supplementary Fig. 11a, both pNPP and BNPP produced yellowish 4-nitrophenol after phosphoester bond cleavage, allowing us to measure the reaction rate quantitatively by recording the absorbance at $400-405 \mathrm{~nm}$. Similar to alkaline phosphatase (ALP), Ce-FMA-FA-20-RT exhibited $\mathrm{pH}$-dependent activity and achieved their maximum activities at pH 10.0 (Supplementary Fig. 12). However, while the activity for BNPP hydrolysis increased with the $\mathrm{pH}$, similar to that for $\mathrm{pNPP}$ hydrolysis, the optimised $\mathrm{pH}$ was 9.0, as shown in Supplementary Fig. 13a. This may be because the rate-limiting step becomes substrate binding when $\mathrm{pH}$ is within 9.0 to 10.0 rather than nucleophilic attack when $\mathrm{pH}$ is within 7.0 to $9.0^{35}$.

Since ALP can convert phosphate compounds (i.e., pyrophosphate) to free phosphate, we continued testing whether CeFMA-FA-20-RT could cleave biological phosphates, such as AMP, ADP, ATP and $\beta$-glycerophosphate $(\beta-G P$ ) (see their structures in Fig. 3a). The molybdenum-blue colorimetric method $^{36}$ was employed to investigate the hydrolysis effect by detecting the Ce-FMA-FA-20-RT-generated free phosphates, as summarised in Supplementary Figure 14. Generally, Ce-FMAFA-20-RT exhibited higher efficiency towards ADP and ATP than AMP and $\beta$-GP, which may be ascribed to more phosphate bonds in ADP and ATP. What is more, alike tendency towards $\mathrm{ADP}$ and ATP was observed, manifesting a "V" shape curve. Intriguingly, though Ce-FMA-FA-20-RT showed weaker activity towards AMP and $\beta$-GP, both the substrates can still be cleaved in neutral environments (HEPES 7.0 and HEPES 7.5, see their conversion rate in Supplementary Table 7), indicating the further possible application in physiological conditions, such as phosphate prodrugs in clinics ${ }^{37}$ and accelerating mineralisation in hard tissue formation due to the supplementary phosphates.
Such success in colorimetric/biological phosphorylated substrates encouraged us next to investigate whether Ce-FMA-FA20-RT could cleave bio-macromolecules with phosphate such as lipid and DNA. We chose cephalin, the second most abundant lipid in living organisms and plasmid DNA as substrates. As shown in Supplementary Figs. 15 and 16, neither of them can be cleaved by Ce-FMA-FA-20-RT. Two reasons could be rationalised: (1) both of the cephalin and plasmid are far larger than the pore aperture of Ce-FMA-FA-20-RT, therefore bringing great steric hindrance for reaction; (2) the long fatty chain in cephalin and large hydrophobic force in DNA may interfere the catalytic reaction.

In summary, this data-informed Ce-FMA-FA-20-RT has robust catalytic activity towards various phosphate molecules (i.e., pNPP, BNPP, AMP, ADP, ATP and $\beta$-GP). The hydrolysis half-life of six MOFs and ALP (Supplementary Fig. 17) are summarised in Supplementary Table 8. Also, a comprehensive heat-map overviewed the hydrolysis action of reported hydrolytic materials as well as Ce-FMA in Fig. 1d. Remarkably, we did not apply the normally required co-catalyst such as polyethylenimine $^{33}$ or $\mathrm{N}$-ethylmorpholine ${ }^{28}$ for this datainformed Ce-FMA MOFs. However, the strong binding affinity between $\mathrm{Zr} / \mathrm{Ce}$ and phosphate ${ }^{38}$ also caused the catalyst poisoning (Supplementary Fig. 18). Future efforts are encouraged to solve the recyclability dilemma for subsequent applications such as organophosphorus antidotes.

Protease-like activity. Given the breadth of biologically and chemically relevant hydrolase reactions and mechanisms, we were curious whether Ce-FMA could mimic other hydrolytic enzymes beyond phosphoesterases. Thus, we next evaluated the catalytic performance of Ce-FMA as a protease. Hydrolysis of amide/ peptide bonds in proteins is more challenging than that of phosphate bonds because the phosphate linkage is more easily cleaved. In contrast to the autolysis of phosphate bond (such as phosphodiester linkage in BNPP, as shown in Supplementary Fig. 13b), the autolysis of amide/peptide bonds has a half-life of 350 years under physiological $\mathrm{pH}$ and temperature ${ }^{39}$.

We applied bovine serum albumin (BSA) as a substrate to test the protease-like activity. BSA is a commonly used and stable 
(a)

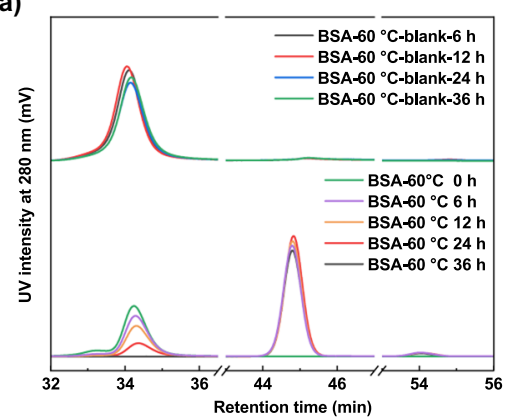

(d)

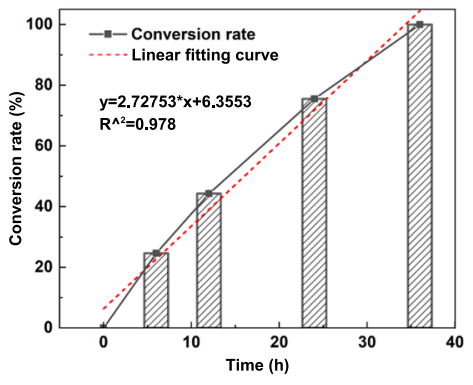

(b)

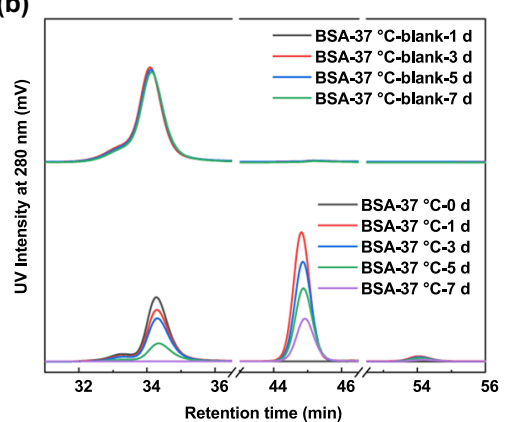

(e)

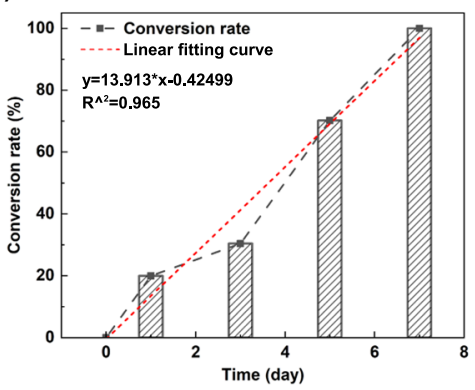

(c)

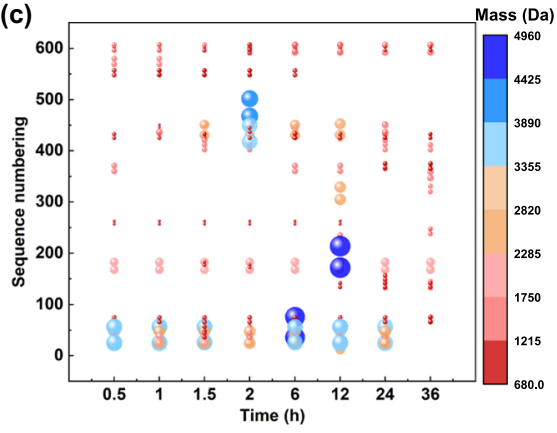

(f)

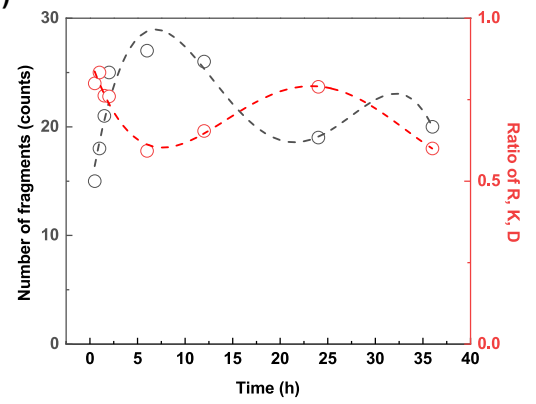

(g)

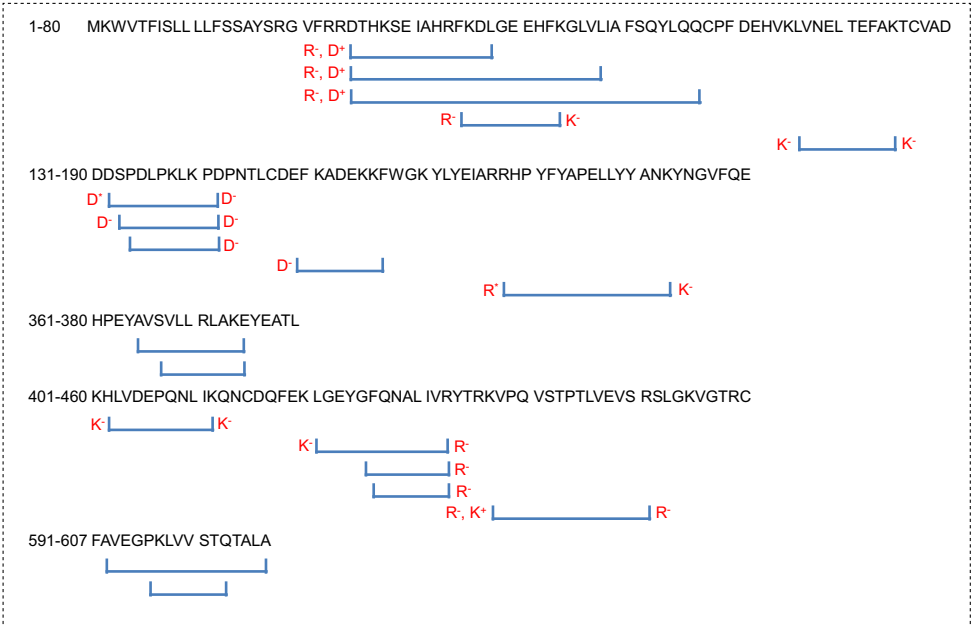

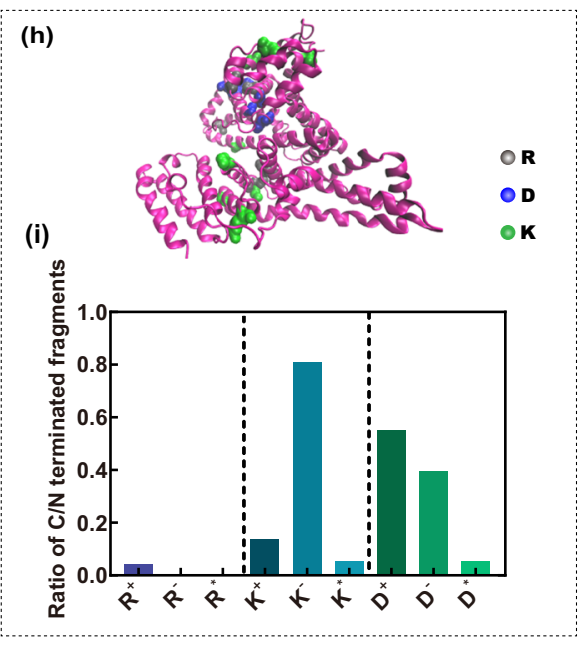

(j)

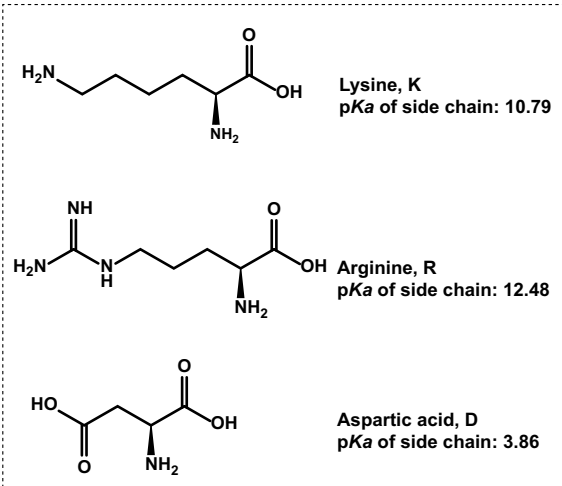

(k)

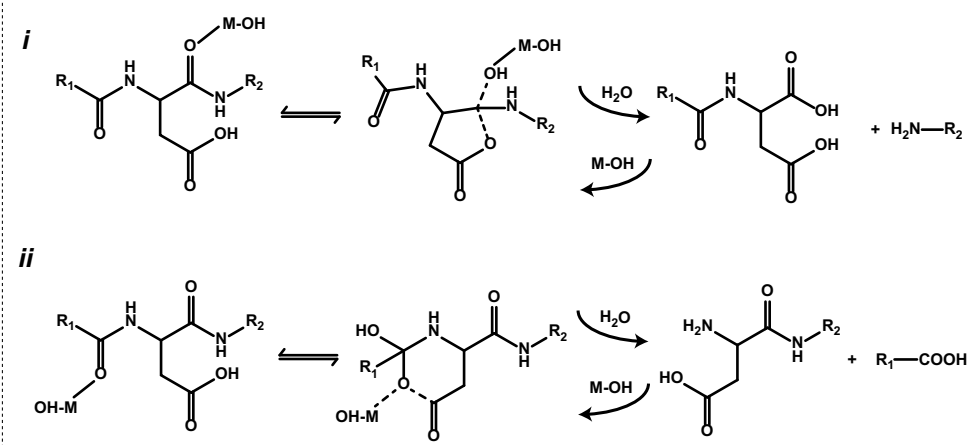

globular protein with 585 amino acids $(66.4 \mathrm{kDa}$ molecular weight). To monitor the hydrolysis of BSA, gel permeation chromatography (GPC) with UV detection at $280 \mathrm{~nm}$ was used. The peak corresponding to BSA at 34-36 min decreased gradually as the reaction proceeded in PBS ( $\mathrm{pH} 7.2-7.4)$ at both $60^{\circ} \mathrm{C}$ and $37^{\circ} \mathrm{C}$, see the lower curves in Fig. $4 \mathrm{a}$, b; while there is no noticeable self-degradation product in the absence of Ce-FMA-
FA-20-RT, see the upper curves in Fig. 4a,b. A 100\% conversion rate was achieved after $36 \mathrm{~h}$ and $7 \mathrm{~d}$ respectively at $60^{\circ} \mathrm{C}$ and $37^{\circ} \mathrm{C}$ in Fig. 4d, e. We then compared the hydrolysis efficiency of our MOF with trypsin (comparison was conducted at $37^{\circ} \mathrm{C}$ ) and MOF-808 (comparison was conducted at $60^{\circ} \mathrm{C}$ ) $\left(\left[\mathrm{Zr}_{6} \mathrm{O}_{4}\left(\mathrm{OH}_{4}(\mathrm{BTC})_{2}-(\mathrm{HCOO})_{6}\right]\right.\right.$ (BTC: benzene-1,3,5-tricarboxylate) because trypsin is a typical protease while MOF- 808 was 
Fig. 4 Protease-like activity of Ce-FMA-FA-20-RT. a GPC profiles of BSA with/without Ce-FMA-FA-20-RT in PBS ( $\mathrm{pH} 7.4$ ) at $60^{\circ} \mathrm{C}$. $\mathbf{b}$ GPC profiles of BSA with/without Ce-FMA-FA-20-RT in PBS (pH 7.4) at $37^{\circ} \mathrm{C}$. c Fragment numbering and mass of fragments of Ce-FMA-FA-20-RT degraded BSA collected at different times. The mass is indicated by the colour gradient and the size of the bubble. $\mathbf{d}$, e Time-dependent conversion rate of BSA corresponding to $\mathbf{a}$ and $\mathbf{b}$, which is calculated by the peak area integral of BSA. $\mathbf{f}$ The number of fragments and ratio of fragments with indicated termini versus reaction time. $\mathbf{g}$ Sequence coverage of BSA and Ce-FMA-FA-20-RT cleaved peptides after $24 \mathrm{~h}$. $\mathbf{h}$ Visualisation of BSA and the surface distribution of cleaved $\mathrm{R}, \mathrm{K}$ and $\mathrm{D}$ after $24 \mathrm{~h}$ at $60^{\circ} \mathrm{C}$ (PDB code: 6QS9, [https://www.pdbus.org/structure/6QS9]). $\mathbf{i}$ Histogram of the frequency distribution of $\mathrm{R}, \mathrm{K}$ and $D$. Specifically, each cleavage site is classified as $N$-terminus $\left(R^{+}, K^{+}\right.$and $\left.D^{+}\right), C$-terminus $\left(R^{-}, K^{-}\right.$and $\left.D^{-}\right)$or both $\left(R^{*}, K^{*}\right.$ and $\left.D^{*}\right)$. $\mathbf{j} C$ hemical structures of $\mathrm{K}, \mathrm{R}$ and $\mathrm{D}$ and the $\mathrm{p} K_{a}$ values of their side chains. $\mathbf{k}$ Mechanisms of cleavage at $\mathrm{D}^{-}(i)$ and $\mathrm{D}^{+}$(ii) termini, showing both of them are thermally stable to form the cyclic anhydride and imide intermediates, adapted from Li et al. ${ }^{52}$.

reported as both good protease-like ${ }^{40}$ and phosphatase-like nanozyme ${ }^{41}$. For trypsin, a conversion rate of $100 \%$ was achieved after $24 \mathrm{~h}$ as shown in Supplementary Fig. 19, demonstrating seven times higher efficiency than Ce-FMA-FA-20-RT $\left(37^{\circ} \mathrm{C}\right.$, 7 days) but 10,000 more costly than nanozyme (Supplementary Table 9). For MOF-808, it achieved no more than $50 \%$ conversion after $24 \mathrm{~h}$, while the conversion of Ce-FMA-FA-20-RT reached $75.54 \%$ (Fig. 4d and Supplementary Fig. 20c, d), even though the surface area of MOF-808 is more than eight times greater than that of Ce-FMA-FA-20-RT (1017.8893 $\mathrm{m}^{2} / \mathrm{g}$ versus $120.4338 \mathrm{~m}^{2} /$ g, respectively, Supplementary Table 6), further confirming the advantage of $\mathrm{Ce}^{4+}$ over $\mathrm{Zr}^{4+}$ in the active site of hydrolytic MOFs.

However, peaks ascribed to degradation products (44-46 min and 53-55 min) were not consistent with the peak of tryptophan (referred as possible final hydrolysis products) which peaked at $93 \mathrm{~min}$ in Supplementary Fig. 21, indicating products with molecular weight larger than tryptophan formed. To obtain the exact molecular weight of the hydrolysed fragments and to identify possible cleavage sites, we further carried out electrospray ionization mass spectrometry (ESI-MS) analysis. As shown in Fig. $4 \mathrm{c}$ and Supplementary Fig. 22, during the process of hydrolysis, the molecular weight of cleaved fragments ranged from 699 to $4764 \mathrm{Da}$, composed of 6 to 41 amino acids. Notably, there were no fragments $>5000 \mathrm{Da}$ even though we collected the sample at the initial process of hydrolysis $(30 \mathrm{~min})$, indicating only small fragments can desorb from Ce-FMA-FA-20-RT. Moreover, the fragments were finally digested into fragments with 6 to 12 amino acids at $36 \mathrm{~h}$, which is alike length with the products digested by natural protease ${ }^{42}$. Coverage of BSA sequence by Ce-FMA-FA-20-RT cleaved peptides from $0.5 \mathrm{~h}$ to $36 \mathrm{~h}$ indicates arginine (R), lysine (K) and aspartic acid (D) are the three main cleavage sites, see Fig. 4f, g and Supplementary Fig. 22. The ratio of selectivity (number of fragments with $\mathrm{R}$ or $\mathrm{K}$ or D/number of total fragments) are plotted in Fig. 4f. Reasonably, the ratio of selectivity decreases when the number of fragments increases but increases if the number of fragments decreases. Moreover, the cleaved sites, plotted in Fig. $4 \mathrm{~h}$, are located on the outer surface of BSA, suggesting a crucial interaction between the outer surface of BSA and Ce-FMA-FA$20-\mathrm{RT}$ which can also be proved by the protein lane of the mixed samples (Supplementary Fig. 23b) and TEM images in Supplementary Fig. $24 \mathrm{e}$, f.

The high protease-like activity of Ce-FMA-FA-20-RT derives from Lewis acid activation mechanism. Similar to the activation of organophosphorus, the Lewis acid (i.e., $\mathrm{Ce}^{4+}$ ) activates carbon by polarising the peptide bond after coordinating with the amide oxygen, thus enhancing the affinity for nucleophile attack to break the amide bond. We ascribed the selective cleavage on sites of $\mathrm{R}, \mathrm{K}$, and $\mathrm{D}$ to two reasons. First, the alkaline/acid groups in side chains accelerate the process of hydrolysis, see in Fig. 4j. Second, hydrolysis of D may undergo two pathways with cyclic anhydride or imide intermediate (Fig. 4k) which are determined by the varied microenvironment of peptides $/ \mathrm{pH}^{43}$. Understanding the specific interaction is helpful for the future design of site- selective protease-like nanozyme and accordingly to acquire potential bioactive peptides. Moreover, since Ce-FMA-FA-20-RT showed different cleavage positions from that by trypsin $(\mathrm{R}, \mathrm{K}$, and $\mathrm{D}$ for Ce-FMA-FA-20-RT vs. $\mathrm{R}$ and $\mathrm{K}$ for trypsin), we further summarised a comparison between Ce-FMA-FA-20-RT and trypsin towards cost, catalytic efficiency and storage in Supplementary Table 9. In conclusion, even though trypsin showed one order of magnitude higher catalytic efficiency than Ce-FMA-FA20-RT, this data-informed nanozyme decreased four order of magnitudes in cost, making itself an optional alternative.

Glycosidase-like activity. The successful demonstration of the ability of Ce-FMA-FA-20-RT to cleave both phosphate bonds and peptide bonds encouraged us to further explore the hydrolytic activity of this material towards glycosidic bonds. However, glycosidase-like nanozymes are rarely studied compared with the other three hydrolase-like nanozymes. To evaluate the broad substrate scope of Ce-FMA-FA-20-RT, we started with chromogenic substrates of 2-nitrophenyl $\beta$-Dgalactopyranoside and 4-nitrophenyl $N$-acetyl- $\beta$-D-glucosaminide (see their structure in Fig. 5a, b). In general, Ce-FMA-FA20 -RT is more efficient to cleave 4 -nitrophenyl $N$-acetyl- $\beta$-Dglucosaminide than 2-nitrophenyl $\beta$-D-galactopyranoside (see in Fig. 5c, d and Supplementary Fig. 25, in which a higher reaction rate was observed in 4-nitrophenyl $N$-acetyl- $\beta$-D-glucosaminide). We further applied an ion chromatography to investigate whether Ce-FMA-FA-20-RT could cleave $\alpha-1,4$ glycosidic bonds in maltose and $\beta-1,4$ glycosidic bonds in lactose when neither of the two substrates have an acetyl amino group. However, there were no cleaved monosaccharide products (Supplementary Fig. 26). These interesting results indicate that the hydrolysis effect on 2-nitrophenyl $\beta$-Dgalactopyranoside and 4 -nitrophenyl $N$-acetyl- $\beta$-D-glucosaminide is benefited by the good leaving groups (i.e., 2-nitrophenyl and 4-nitrophenyl groups). Therefore, we continued choosing carboxymethyl chitosan as a substrate since the carboxymethyl groups may coordinate with $\mathrm{Ce}$ clusters which may help decrease the adsorption energy. GPC with a refractive index detector (RID) was used to measure the product of carboxymethyl chitosan. The results of GPC demonstrate that CeFMA-FA-20-RT is able to cleave carboxymethyl chitosan in alkaline ( $\mathrm{pH} 8.0$ ) environments both at $37^{\circ} \mathrm{C}$ and $60^{\circ} \mathrm{C}$, see Fig. $5 \mathrm{e}$. The area of the peak ascribed to product increased with the temperature enhanced and the product peak in $60^{\circ} \mathrm{C}$ shifted more right than that in $37^{\circ} \mathrm{C}$ (retention time $42-44 \mathrm{~min}$ vs. 40-42 min). Moreover, the UV intensity peak at $254 \mathrm{~nm}$ to product grows more sharp and intense, as outlined by the dashed blue rectangle in Supplementary Fig. 27, indicating the successful cleavage towards carboxymethyl chitosan. We also applied carboxymethyl chitosan to test the reusability of CeFMA-FA-20-RT. As shown in Supplementary Fig. 28, Ce-FMAFA-20 remained $80 \%$ activity in the second recycle but decreased dramatically in the third recycle. 

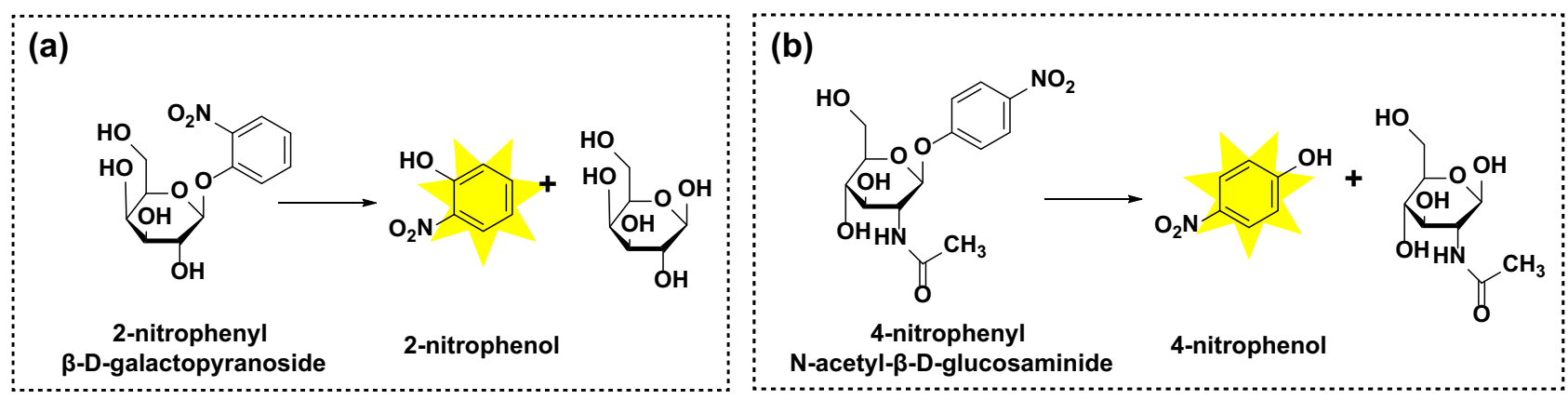

(c)

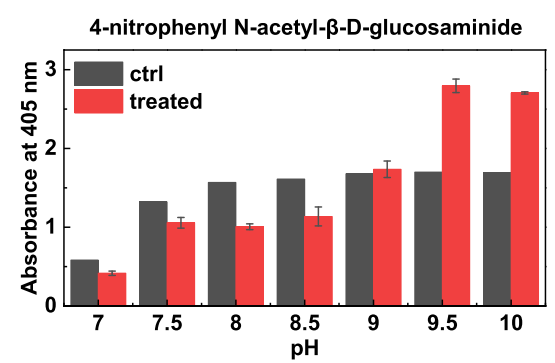

(e)

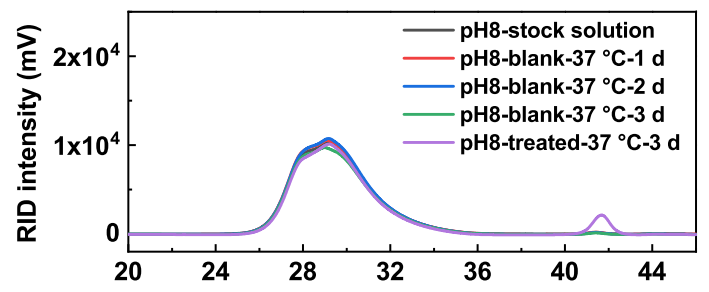

(d)
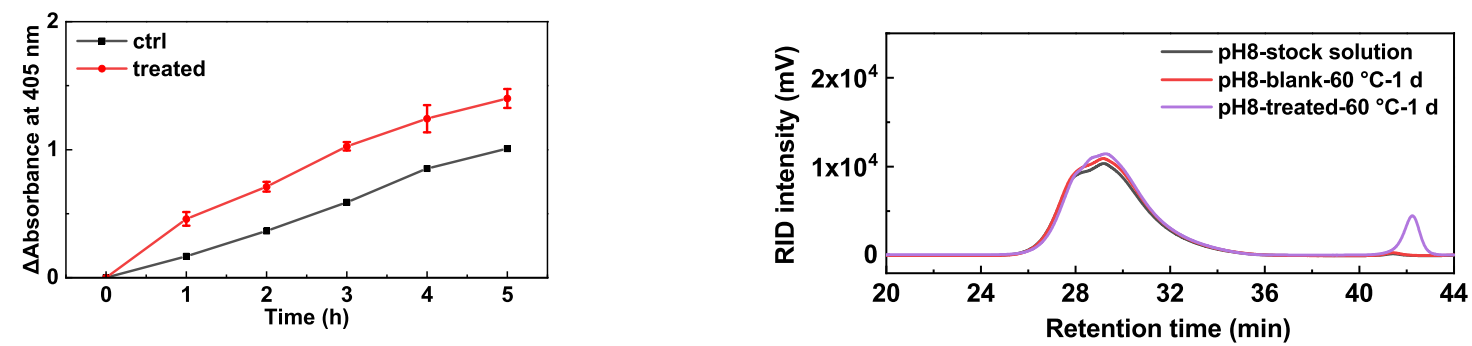

Fig. 5 Glycosidase-like activity of Ce-FMA-FA-20-RT. a and $\mathbf{b}$ Schematic illustration of hydrolysis and the detection principle of 2-nitrophenyl $\beta$-Dgalactopyranoside in $\mathbf{a}$ and 4-nitrophenyl $\mathrm{N}$-acetyl- $\beta$-D-glucosaminide in $\mathbf{b}$. c The optical density at $405 \mathrm{~nm}$ of hydrolysed product of 4 -nitrophenyl $\mathrm{N}$-acetyl- $\beta$-Dglucosaminide under different $\mathrm{pHs}$ at $60^{\circ} \mathrm{C}$ for $8 \mathrm{~h}$. Data are presented as mean \pm standard error of the mean $(n=3)$. d Optical density change at $405 \mathrm{~nm}$ of hydrolysed product of 4 -nitrophenyl $\mathrm{N}$-acetyl- $\beta$-D-glucosaminide under $\mathrm{pH} 10.0$ bathed at $60^{\circ} \mathrm{C}$ for $5 \mathrm{~h}$. ( $\Delta \mathrm{Absorbance}=\mathrm{Absorbance}_{\text {time }}-\mathrm{Absorbance}_{\mathrm{O}} \mathrm{h}$ ) Data are presented as mean \pm standard error of the mean $(n=3)$. e GPC profiles of carboxymethyl chitosan before and after treatment with $\mathrm{Ce}-\mathrm{FMA}-\mathrm{FA}-20-\mathrm{RT}$ at $37^{\circ} \mathrm{C}$ or $60^{\circ} \mathrm{C}$ under $\mathrm{pH} 8.0$.

Generally, Ce-FMA-FA-20-RT prefers to cleaving glucuronide derivatives with (1) groups lowering the adsorption energy; (2) groups lowering the desorption energy (such as 2-nitrophenyl and 4-nitrophenyl). More universal and powerful glycosidase-like nanozyme still needs further improvement.

Application of Ce-FMA-FA-20-RT in hydrolysis of multiple substrates for biofilm degradation. As described above, CeFMA-FA-20-RT has been shown to hydrolyse several individual substrates containing phosphoester bonds, amide/peptide bonds and glycosidic bonds. A more challenging feat is the degradation of mixture of these substrates, such as biofilms. A biofilm is a microbial consortium with self-produced extracellular polymeric substances (EPS). Inside the three-dimensional architecture of a biofilm, the EPS forms a scaffold that hosts the bacteria and is responsible for external defence, adhesion to surfaces, connectivity and nutrient trapping. Due to the roles that a biofilm plays as a protector and energy supplier, cells in a biofilm have adopted properties different from those of planktonic bacteria, thus limiting the efficacy of antimicrobials against bacterial infection involving biofilms ${ }^{44}$. One of the reasons why biofilms are so difficult to deal with is their varied compositions: polysaccharides/ phosphoethanolamine cellulose ${ }^{45}$, proteins, nucleic acids and lipids (Fig. 6a). This complexity increases the difficulty for a single enzyme to disrupt a biofilm. In this regard, Ce-FMA-FA-20-RT, with multiple hydrolytic activities, may be advantageous, as it has been proven to be effective in cleaving phosphoester bonds, amide/peptide bonds and glycosidic bonds, which all exist in biofilms in various forms.

To test this hypothesis, we applied two representative biofilms formed by gram-negative bacteria (E. coli) and gram-positive bacteria (S. aureus). Bacterial cells were grown for $48 \mathrm{~h}$ in a 24 -well plate stationarily to form biofilms. Then, Ce-FMA-FA-20-RT together with fresh medium was added, followed by another $12 \mathrm{~h}$ incubation at $37^{\circ} \mathrm{C}$ to allow catalytic hydrolysis of the biofilms. Both of the scanning electronic microscope (SEM) images and confocal laser scanning microscope (CLSM) images in Fig. 6b, d display the expected thick, bulk-like adhesions between cells when no Ce-FMAFA-20-RT treatment was applied, demonstrating the successful formation of biofilms. In contrast, the adhesion among cells became tenuous and fibre-like after Ce-FMA-FA-20-RT treatment, and clear gaps were also observed. Crystal violet staining assay was applied to semi-quantitate the amount of biofilm. As shown in Fig. 6c, e, a significant difference was observed before and after Ce-FMA-FA-20RT treatment in both gram-negative bacteria (E. coli) and grampositive bacteria (S. aureus). Moreover, plates spread with Ce-FMAFA-20-RT-treated E. coli and S. aureus indicated negligible adverse effects on bacterial growth, as shown in Supplementary Fig. 29. This finding is also consistent with the intact cell morphology shown in Fig. 6b, d after treatment with Ce-FMA-FA-20-RT, confirming that the decrease in biofilm formation could only be attributed to hydrolysis rather than to bacterial cell death. 


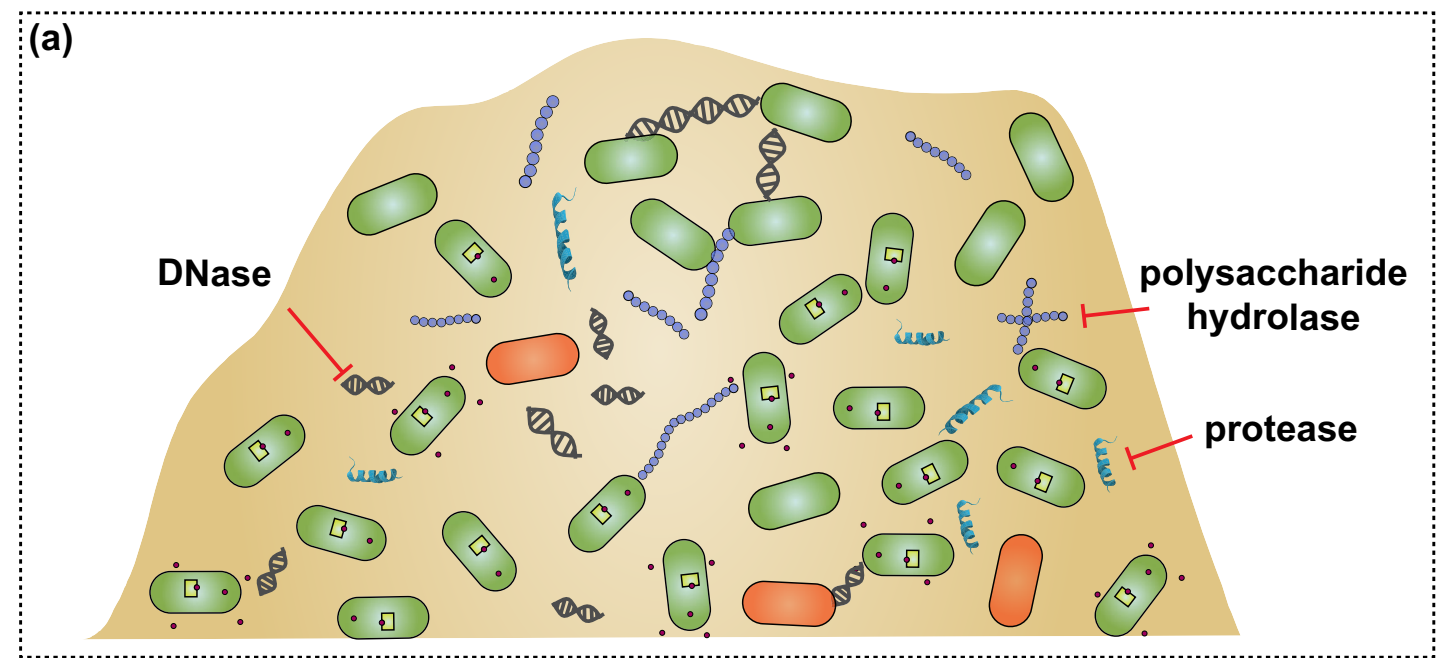

(b)
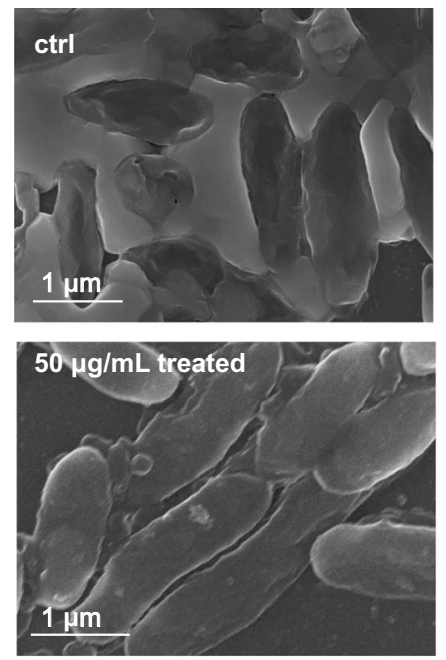

(d)
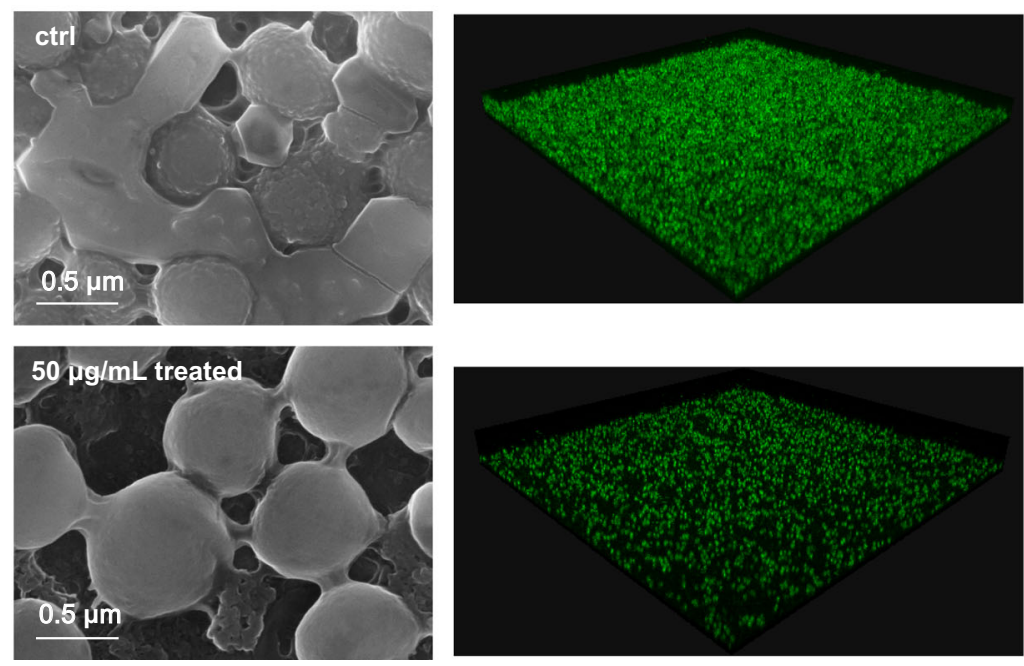

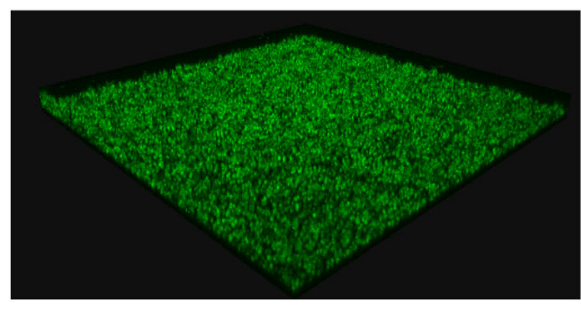

(e)
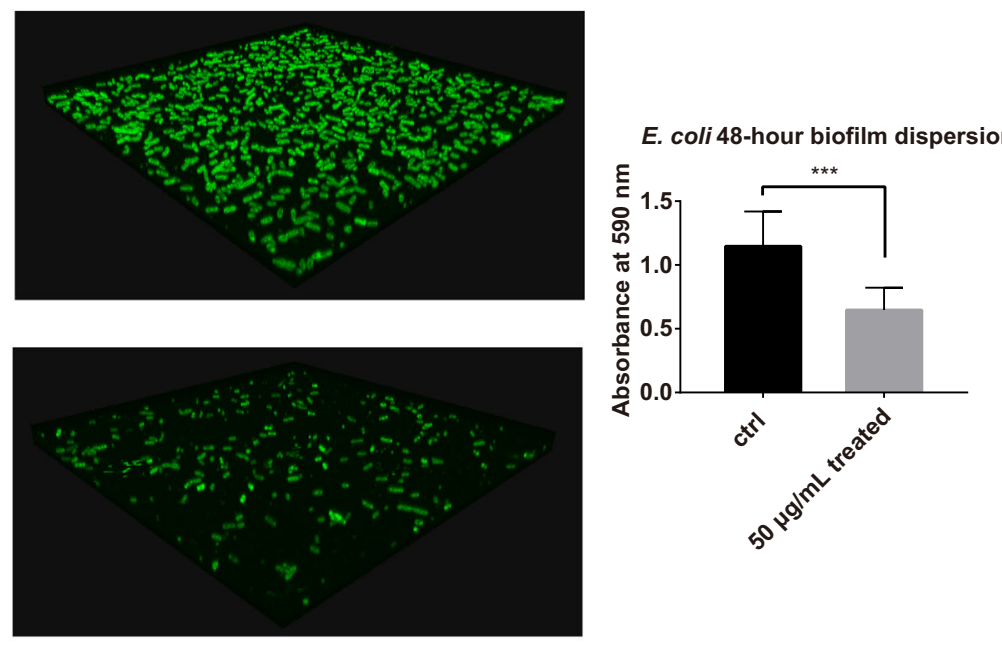

(e)

S. aureus 48 -hour biofilm dispersio

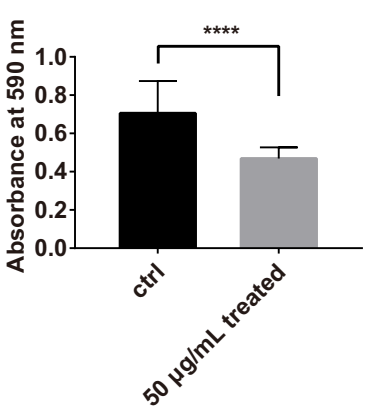

\section{Discussion}

In conclusion, we introduced a data-informed approach to discover a high-performance hydrolytic nanozyme by systematically analysing 105 published papers to supplement the underdeveloped field of research on hydrolytic nanozymes. Analysis of these data indicates that MOF is a good scaffold to embed hydrolytic active sites for their tuneable metal clusters and ligands. Further structured data suggests that Lewis acidity of metal clusters in MOFs and the density of active sites which is adjusted by ligand are two critical elements. Consequently, we screened $\mathrm{Ce}^{4+}$ as a promising metal ion component and applied a short ligand, FMA, to construct a UiO-66-like MOF. 
Fig. 6 Hydrolytic performance of Ce-FMA-FA-20-RT on biofilms. a Schematic of the diverse biofilm components and strategies to combat biofilm formation by using hydrolytic enzymes. b SEM and confocal images of biofilms formed by E. coli without/with Ce-FMA-FA-20-RT treatment (upper: low magnification, lower: high magnification). The biofilm was stained by FilmTracer ${ }^{\mathrm{TM}} \mathrm{FM}^{\mathrm{TM}}$ 1-43 Green Biofilm Cell Stain. And the confocal images were obtained using an Olympus confocal microscope FV 3000 and a $\times 100 / 1.45$ NA oil immersion objective. c Crystal violet staining of biofilms formed by E. coli without/with Ce-FMA-FA-20-RT treatment (significance level: ${ }^{\star \star \star} P<0.001$ ). Data are presented as the mean \pm standard error after removing the maximum and minimum, $n=12$. d SEM and confocal images of biofilms formed by S. aureus without/with Ce-FMA-FA-20-RT treatment (upper: low magnification, lower: high magnification). e Crystal violet staining of biofilms formed by S. aureus without/with Ce-FMA-FA-20-RT treatment (significance level: $\left.{ }^{\star \star \star \star} P<0.0001\right)$. Data are presented as the mean \pm standard error after removing the maximum and minimum, $n=16$.

Encouragingly, this rationally designed Ce-FMA was highly efficient in hydrolysing a broad scope of substrates and exhibited huge promise to be further developed. First, nonactivated CeFMA exhibited excellent phosphatase-like activity (half-life of $<2 \mathrm{~min}$ ) even without the use of co-catalysts. Second, Ce-FMA showed high activity towards BSA hydrolysis, with 12.7 times better efficiency than the multi-functional Zr-based MOF-808. The new cleavage sites (such as D sites) brought by nanozymes expand the diversity of peptides and offer additional probability to obtain active peptides. Third, we evaluated the ability of this Ce-FMA MOF to cleave glycosidic bonds in selected substrates. Last, we applied Ce-FMA to a mixture of biomacromolecules, i.e., biofilms, from both gram-negative bacteria (E. coli) and grampositive bacteria (S. aureus), which is hopefully applied in biomedicine and marine. Additionally, this data-informed approach can be applied to deduce abiotic active sites rather than being limited to the direct modification of natural active sites.

We acknowledge that more chemical information about hydrolytic MOF nanozymes should be analysed, such as the topology of the coordination network, pore aperture to the substrates and the modification of functional groups. However, due to the limited amount of available data, the current study was not suitable for machine learning or chemoinformatic models with multiple variables and was unable to discover esterase mimics. Nevertheless, given the rapid development of this field, we anticipate that machine learning as well as other artificial intelligence techniques will be further leveraged for the design and discovery of new nanozymes, such as powerful esterase mimics for degradation of PET (polyethylene terephthalate) in the near future.

\section{Methods \\ Synthesis of Zr-FMA. Zr-FMA was synthesised as previously reported ${ }^{46}$. For details, FMA (116 mg, $1 \mathrm{mmol})$ and $\mathrm{ZrOCl}_{2} \cdot 8 \mathrm{H}_{2} \mathrm{O}(322.25 \mathrm{mg}, 1 \mathrm{mmol})$ were suspended in $10 \mathrm{~mL}$ of water/formic acid mixed solution (water, $9.8 \mathrm{~mL}$; formic acid, $0.2 \mathrm{~mL}$ ) and heated to $105^{\circ} \mathrm{C}$, refluxing for $24 \mathrm{~h}$. The obtained white powder product was centrifuged $(4100 \times g, 1 \mathrm{~min})$ and washed with water for three times. The obtained solids were dried in a vacuum oven at $60^{\circ} \mathrm{C}$ overnight.}

Synthesis of Hf-FMA. Hf-FMA was synthesised using the same method as $\mathrm{Zr}$ FMA, except changing $\mathrm{ZrOCl}_{2} \cdot 8 \mathrm{H}_{2} \mathrm{O}$ into $\mathrm{HfCl}_{4}(320 \mathrm{mg}, 1 \mathrm{mmol})$.

Synthesis of Ce-FMA. Typically, FMA $(1.74 \mathrm{~g}, 15 \mathrm{mmol})$ and $\mathrm{Ce}\left(\mathrm{NH}_{4}\right)_{2}\left(\mathrm{NO}_{3}\right)_{2}$ $(1.6447 \mathrm{~g}, 15 \mathrm{mmol})$ were suspended in $30 \mathrm{~mL}$ solution with water and modulators (see their ratio in Supplementary Table 5), stiring for $10 \mathrm{~min}$ at room temperature. The yellowish solution was centrifuged $(4100 \times g, 1 \mathrm{~min})$ and washed with water and ethanol three times. Then, the obtained solids were dried in vacuum oven at $60^{\circ} \mathrm{C}$ overnight.

Synthesis of UiO (University of Oslo)-66 (Zr). BDC (124.5 mg, $0.75 \mathrm{mmol})$ and $\mathrm{ZrOCl}_{2} \cdot 8 \mathrm{H}_{2} \mathrm{O}(241.69 \mathrm{mg}, 0.75 \mathrm{mmol})$ were suspended in $30 \mathrm{~mL}$ of $\mathrm{DMF} /$ formic acid mixed solution (DMF, $27 \mathrm{~mL}$; formic acid, $3 \mathrm{~mL}$ ). After sonication for $5 \mathrm{~min}$, the mixture was transferred into a $50 \mathrm{~mL}$ Teflon-lined autoclave and heated to $120^{\circ} \mathrm{C}$ for $24 \mathrm{~h}$. The obtained white powder product was centrifuged $(4100 \times g$, $3 \mathrm{~min}$ ) and washed with water twice and then with acetone twice. Then the obtained solids were dried in vacuum oven at $60^{\circ} \mathrm{C}$ overnight.
Synthesis of UiO-66 (Hf). BDC (124.5 mg, $0.75 \mathrm{mmol})$ and $\mathrm{HfCl}_{4}(240 \mathrm{mg}$, $0.75 \mathrm{mmol}$ ) were suspended in $30 \mathrm{~mL}$ of DMF/formic acid mixed solution (DMF $27 \mathrm{~mL}$; formic acid, $3 \mathrm{~mL}$ ). After sonication for $5 \mathrm{~min}$, the mixture was transferred into a $50 \mathrm{~mL}$ Teflon-lined autoclave and heated to $123^{\circ} \mathrm{C}$ for $40 \mathrm{~h}$. The obtained white powder product was centrifuged $(4100 \times \mathrm{g}, 3 \mathrm{~min})$ and washed with water twice and then with acetone twice. Then the obtained solids were dried in vacuum oven at $60^{\circ} \mathrm{C}$ overnight.

Synthesis of UiO-66 (Ce). UiO-66 (Ce) was synthesised as previously reported with minor modification ${ }^{31,33}$. In detail, an ultrapure water solution of $\mathrm{Ce}(\mathrm{N}$ $\left.\mathrm{H}_{4}\right)_{2}\left(\mathrm{NO}_{3}\right)_{6}(0.533 \mathrm{M}, 2.4 \mathrm{~mL})$ was added into $7.5 \mathrm{~mL}$ of DMF solution containing $212.4 \mathrm{mg}$ BDC. The mixture was then refluxed in an oil bath at $102^{\circ} \mathrm{C}$ for $15 \mathrm{~min}$. Product was then centrifuged $(2010 \times g, 1 \mathrm{~min})$ and resuspended in fresh DMF for $1 \mathrm{~h}$. After 3 cycles of centrifugation and soaking in fresh DMF for $1 \mathrm{~h}$, the solids were washed by ethanol for three times. Finally, the yellowish powder product was placed in vacuum oven at $60^{\circ} \mathrm{C}$ overnight to remove solvent. To activate UiO-66, the solid was soaked in ethanol overnight to fully exchange solvent and then collected by centrifuged $(4100 \times \mathrm{g}, 3 \mathrm{~min})$ and dried at $100^{\circ} \mathrm{C}$ in vacuum oven for more than $12 \mathrm{~h}$.

Synthesis of MOF-808. MOF- 808 was synthesised as previously reported ${ }^{40}$. In details, $\mathrm{H}_{3} \mathrm{BTD}(157.2 \mathrm{mg}, 0.75 \mathrm{mmol})$ along with $\mathrm{ZrOCl}_{2} .8 \mathrm{H}_{2} \mathrm{O}(241.8 \mathrm{mg}, 0.75$ $\mathrm{mmol}$ ) was added into the mixture solution of $30 \mathrm{~mL}$ of DMF and $30 \mathrm{~mL} \mathrm{FA}$ in a $100 \mathrm{~mL}$ flask. The solution was then refluxed in an oil bath at $130{ }^{\circ} \mathrm{C}$ for $48 \mathrm{~h}$ Product was washed by DMF for one time and then washed by ethanol for several times. Finally, the white powder product was placed in vacuum oven at $70^{\circ} \mathrm{C}$ overnight to remove solvent.

\section{Optimisation on synthetic conditions of Ce-FMAs by comparing ALP-like}

activity. Typically, the activity assays were conducted in the optimised $\mathrm{pH} 10.0$ with a fixed concentration of $10 \mathrm{mM} \mathrm{pNPP}$ and $0.5 \mathrm{mg} / \mathrm{mL}$ catalyst prepared at different conditions with 8 parallel samples. After incubation for $20 \mathrm{~min}$ at $37^{\circ} \mathrm{C}$, the absorbance at $400 \mathrm{~nm}$ was recorded by Tecan Pro 200 Microplate Reader for mass activity comparison. The specific activity comparison was normalised by BET surface area in Supplementary Table 6. Due to the presence of high-energy phosphate bond in pNPP, all the data have been treated to subtract the autolysis of pNPP and the background of catalysts. The maximum and minimum were removed when plotting.

Optimisation on synthetic conditions of Ce-FMA by comparing catalytic activity. The optimisation of synthetic conditions was conducted by monitoring the absorbance at $400 \mathrm{~nm}$. Typically, the activity assay was conducted in the optimised pH 9.0 with a fixed concentration of $0.8 \mathrm{mM}$ BNPP and $0.5 \mathrm{mg} / \mathrm{mL}$ catalyst prepared at different conditions with at least 3 parallel samples. After incubation for $3 \mathrm{~h}$ at $37^{\circ} \mathrm{C}$, the absorbance at $400 \mathrm{~nm}$ was recorded by Tecan Pro 200 Microplate Reader for mass activity comparison. The specific activity comparison was normalised by BET surface area in Supplementary Table 6. Due to the presence of high-energy phosphate bond in BNPP, all the data have been treated to subtract the autolysis of BNPP and the background of catalysts.

GPC monitor of the BSA degradation process. BSA $(66.4 \mathrm{kDa})$ was chosen as a model to test whether Ce-FMA-FA-20-RT could cleavage peptide bonds. Typically, $2 \mathrm{mg} / \mathrm{mL}$ BSA mixed with $5 \mathrm{mg} / \mathrm{mL}$ Ce-FMA-FA-20-RT in $1 \times$ PBS buffer was bathed at $60^{\circ} \mathrm{C}$ or $37^{\circ} \mathrm{C}$ with stirring at $1200 \mathrm{rpm}$. Equivalent reaction mixture was taken at $6 \mathrm{~h}, 12 \mathrm{~h}, 24 \mathrm{~h}, 36 \mathrm{~h}$ and $48 \mathrm{~h}$ for $60^{\circ} \mathrm{C}$ group and $1 \mathrm{~d}, 3 \mathrm{~d}, 5 \mathrm{~d}$ and $7 \mathrm{~d}$ for $37^{\circ} \mathrm{C}$ group. The mixture was centrifuged and supernatant was collected and diluted 4 times before being filtered with $0.22 \mu \mathrm{m}$ filter. GPC profiles were monitored by UV detector at $280 \mathrm{~nm}$. The conversion rate was defined by the decreased area on the peak of original BSA. The conversion rate $\mu$ could be described as follow:

$$
\mu=1-\frac{\text { area of BSA after hydrolysis }}{\text { area of BSA before hydrolysis }} \times 100 \%
$$

ESI-MS characterisation on hydrolytic fragments of BSA. Hydrolysed products taken out at $0.5 \mathrm{~h}, 1.0 \mathrm{~h}, 1.5 \mathrm{~h}, 2.0 \mathrm{~h}, 6.0 \mathrm{~h}, 12 \mathrm{~h}, 24 \mathrm{~h}$ and $36 \mathrm{~h}$ were then centrifuged 
to collect the supernatants. Before test, samples were diluted to 10 times by ultrapure water and removed salt by stage tip, then lyophilised and re-suspended in water for ESI-MS

Hydrolytic effect on $\boldsymbol{\beta}$-glycosidic bond. 2-nitrophenyl $\beta$-D-galactopyranoside was chosen to study the cleavage effect in different buffers. Typically, $200 \mu \mathrm{L}$ of solutions containing $5 \mathrm{mM} 2$-nitrophenyl $\beta$-D-galactopyranoside and $1 \mathrm{mg} / \mathrm{mL} \mathrm{Ce}$ FMA-FA-RT-20 were incubated in different buffers at $60^{\circ} \mathrm{C}$ for $8 \mathrm{~h}$. Three parallel samples were conducted each time. After $8 \mathrm{~h}, 180 \mu \mathrm{L}$ of the solution was added into 96-well plate and measured by Tecan Pro 200 Microplate Reader at $420 \mathrm{~nm}$

Hydrolytic effect on $\boldsymbol{\beta}$ - $\mathbf{N}$-acetyl-glycosidic bond. 4-nitrophenyl $N$-acetyl- $\beta$-Dglucosaminide was chosen to study the cleavage effect in different buffers. Typically, $200 \mu \mathrm{L}$ of solutions containing $5 \mathrm{mM} 4$-nitrophenyl $N$-acetyl- $\beta$-D-glucosaminide and $1 \mathrm{mg} / \mathrm{mL}$ Ce-FMA-FA-RT-20 were incubated in different buffers at $60^{\circ} \mathrm{C}$ for $8 \mathrm{~h}$. Three parallel samples were conducted each time. After $8 \mathrm{~h}, 180 \mu \mathrm{L}$ of the solution was added into 96-well plate and measured by Tecan Pro 200 Microplate Reader at $405 \mathrm{~nm}$.

\begin{abstract}
Hydrolysis of $\beta-1-4$ glycosidic bond (carboxymethyl chitosan as substrate in alkaline environment). Since carboxymethyl chitosan tends to dissolve in neutral or alkaline solution, carboxymethyl chitosan was chosen to study the degradation effect in alkaline (Tris-HCl buffer at 8.0) environment. Typically, $1 \mathrm{~mL}$ of $1.2 \% \mathrm{wt}$ carboxymethyl chitosan (dissolved in buffer) was mixed with $200 \mu \mathrm{L}$ of $10 \mathrm{mg} / \mathrm{mL}$ Ce-FMA-FA-20-RT in constant temperature oscillator at $37^{\circ} \mathrm{C}$ with shaking $(220 \mathrm{rpm})$. Three parallel samples were conducted each time. After $24 \mathrm{~h}$, the mixture was centrifuged and then filtered with $0.22 \mu \mathrm{m}$ filter for GPC test by RID detector and Pullulan was applied as an internal reference (P5 for carboxymethyl chitosan, respectively)
\end{abstract}

SEM observation on the dispersion effect of formed biofilm. In all, 24-well plate covered with Nest circle microscope cover glass was used to incubate bacteria. After $48 \mathrm{~h}$ development of biofilm, $2 \mathrm{~mL}$ of culture medium with or without $50 \mu \mathrm{g} / \mathrm{mL}$ Ce-FMA-FA-20-RT was added to study whether Ce-FMA-FA-20-RT could hydrolyse existed biofilm at $37^{\circ} \mathrm{C}$. After $12 \mathrm{~h}$, the medium was discarded and the wells were washed by PBS three times gently to remove suspended bacteria. Remained biofilm was then fixed by methanol for $15 \mathrm{~min}$. Next, the cover glass was sputtered with $\mathrm{Cr}$ for subsequent SEM observation.

CLSM observation on the dispersion effect of formed biofilm. Biofilm was cultured in coverglass bottom dish $(10 \mathrm{~mm})$ for $48 \mathrm{~h}$ and then treated with/without the addition of $50 \mu \mathrm{g} / \mathrm{mL}$ Ce-FMA-FA- 20 for $12 \mathrm{~h}$ at $37^{\circ} \mathrm{C}$. Then, the medium was discarded and the wells were washed by PBS three times gently to remove suspended bacteria. The biofilm was stained by FilmTracer ${ }^{\mathrm{mm}} \mathrm{FM}^{\mathrm{mm}} 1-43$ Green Biofilm Cell Stain. And the confocal images were obtained using an Olympus confocal microscope FV 3000 and a $\times 100 / 1.45$ NA oil immersion objective.

Crystal violet staining of biofilm. The biofilm dispersion was similar as above without covering Nest circle microscope cover glass in 24-well plate. After fixation, wells were stained with $150 \mu \mathrm{L}$ of $0.1 \%_{\text {wt }}$ crystal violet for $10 \mathrm{~min}$ and then washed with $200 \mu \mathrm{L}$ of water to remove floating colour. After drying, $250 \mu \mathrm{L}$ of ethanol was used to elute the crystal violet of each well. The stained solution was diluted moderately in 96 -well plate for further measure at the absorbance at $590 \mathrm{~nm}$ by Tecan Pro 200 Microplate Reader.

\section{Data availability}

The data generated in this study are provided in the Supplementary Information/Source Data file. Source data are provided with this paper.

Received: 15 January 2021; Accepted: 15 December 2021; Published online: 11 February 2022

\section{References}

1. Mirts, E. N., Petrik, I. D., Hosseinzadeh, P., Nilges, M. J. \& Lu, Y. A designed heme-[4Fe-4S] metalloenzyme catalyzes sulfite reduction like the native enzyme. Science 361, 1098-1101 (2018).

2. Grommet, A. B., Feller, M. \& Klajn, R. Chemical reactivity under nanoconfinement. Nat. Nanotechnol. 15, 256-271 (2020).

3. $\mathrm{Wu}, \mathrm{J}$. et al. Nanomaterials with enzyme-like characteristics (nanozymes) next-generation artificial enzymes (II). Chem. Soc. Rev. 48, 1004-1076 (2019).

4. Gao, L. et al. Intrinsic peroxidase-like activity of ferromagnetic nanoparticles. Nat. Nanotechnol. 2, 577-583 (2007).
5. Manea, F., Houillon, F. B., Pasquato, L. \& Scrimin, P. Nanozymes: goldnanoparticle-based transphosphorylation catalysts. Angew. Chem. Int. Ed. 43, 6165-6169 (2004)

6. Huang, Y., Ren, J. \& Qu, X. Nanozymes: classification, catalytic mechanisms, activity regulation, and applications. Chem. Rev. 119, 4357-4412 (2019).

7. Liang, M. \& Yan, X. Nanozymes: from new concepts, mechanisms, and standards to applications. Acc. Chem. Res. 52, 2190-2200 (2019).

8. Wei, $\mathrm{H}$. \& Wang, $\mathrm{E} . \mathrm{Fe}_{3} \mathrm{O}_{4}$ magnetic nanoparticles as peroxidase mimetics and their applications in $\mathrm{H}_{2} \mathrm{O}_{2}$ and glucose detection. Anal. Chem. 80, 2250-2254 (2008).

9. Zhang, Z., Zhang, X., Liu, B. \& Liu, J. Molecular imprinting on inorganic nanozymes for hundred-fold enzyme specificity. J. Am. Chem. Soc. 139, 5412-5419 (2017).

10. Gupta, A., Das, R., Yesilbag Tonga, G., Mizuhara, T. \& Rotello, V. M. Chargeswitchable nanozymes for bioorthogonal imaging of biofilm-associated infections. ACS Nano 12, 89-94 (2018).

11. Kim, J. et al. Continuous $\mathrm{O}_{2}$-evolving $\mathrm{MnFe}_{2} \mathrm{O}_{4}$ nanoparticle-anchored mesoporous silica nanoparticles for efficient photodynamic therapy in hypoxic cancer. J. Am. Chem. Soc. 139, 10992-10995 (2017).

12. Mandoli, C. et al. Stem cell aligned growth induced by $\mathrm{CeO}_{2}$ nanoparticles in PLGA scaffolds with improved bioactivity for regenerative medicine. $A d v$. Funct. Mater. 20, 1617-1624 (2010).

13. Singh, B. K. Organophosphorus-degrading bacteria: ecology and industrial applications. Nat. Rev. Microbiol. 7, 156-164 (2009).

14. Xia, M. et al. Assembly of the active center of organophosphorus hydrolase in metal-organic frameworks via rational combination of functional ligands. Chem. Commun. 53, 11302-11305 (2017).

15. Jin, C., Zhang, S., Zhang, Z. \& Chen, Y. Mimic carbonic anhydrase using metal-organic frameworks for $\mathrm{CO}_{2}$ capture and conversion. Inorg. Chem. 57, 2169-2174 (2018)

16. Pieters, G., Pezzato, C. \& Prins, L. J. Controlling supramolecular complex formation on the surface of a monolayer-protected gold nanoparticle in water. Langmuir 29, 7180-7185 (2013).

17. Chen, J. et al. Bio-inspired nanozyme: a hydratase mimic in a zeolitic imidazolate framework. Nanoscale 11, 5960-5966 (2019).

18. Maiti, S., Fortunati, I., Ferrante, C., Scrimin, P. \& Prins, L. J. Dissipative selfassembly of vesicular nanoreactors. Nat. Chem. 8, 725-731 (2016).

19. Sun, M. et al. Site-selective photoinduced cleavage and profiling of DNA by chiral semiconductor nanoparticles. Nat. Chem. 10, 821-830 (2018).

20. Vaissier Welborn, V. \& Head-Gordon, T. Computational design of synthetic enzymes. Chem. Rev. 119, 6613-6630 (2019).

21. Key, H. M., Dydio, P., Clark, D. S. \& Hartwig, J. F. Abiological catalysis by artificial haem proteins containing noble metals in place of iron. Nature $\mathbf{5 3 4}$ 534-537 (2016)

22. Zhou, Z. \& Roelfes, G. Synergistic catalysis in an artificial enzyme by simultaneous action of two abiological catalytic sites. Nat. Catal. 3, 289-294 (2020).

23. Wilhelm, S. et al. Analysis of nanoparticle delivery to tumours. Nat. Rev. Mater. 1, 16014 (2016).

24. Son, F. A. et al. Uncovering the role of metal-organic framework topology on the capture and reactivity of chemical warfare agents. Chem. Mater. 32, 4609-4617 (2020).

25. Yaghi, O. M. et al. Reticular synthesis and the design of new materials. Nature 423, 705-714 (2003).

26. Zhao, M. et al. Metal-organic frameworks as selectivity regulators for hydrogenation reactions. Nature 539, 76-80 (2016).

27. Horike, S., Shimomura, S. \& Kitagawa, S. Soft porous crystals. Nat. Chem. 1, 695-704 (2009).

28. Mondloch, J. E. et al. Destruction of chemical warfare agents using metal-organic frameworks. Nat. Mater. 14, 512-516 (2015).

29. Liu, L. et al. Imaging defects and their evolution in a metal-organic framework at sub-unit-cell resolution. Nat. Chem. 11, 622-628 (2019).

30. Wang, S., McGuirk, C. M., d'Aquino, A., Mason, J. A. \& Mirkin, C. A. Metal-organic framework nanoparticles. Adv. Mater. 30, e1800202 (2018)

31. Lammert, M. et al. Cerium-based metal organic frameworks with UiO-66 architecture: synthesis, properties and redox catalytic activity. Chem. Commun. 51, 12578-12581 (2015).

32. Yang, P., Zhuang, Q., Li, Y. \& Gu, J. Green separation of rare earth elements by valence-selective crystallization of MOFs. Chem. Commun. 55, 14902-14905 (2019).

33. Islamoglu, T. et al. Cerium(IV) vs zirconium(IV) based metal-organic frameworks for detoxification of a nerve agent. Chem. Mater. 29, 2672-2675 (2017).

34. Plonka, A. M. et al. In situ probes of capture and decomposition of chemical warfare agent simulants by Zr-based metal organic frameworks. J. Am. Chem. Soc. 139, 599-602 (2017) 
35. Katz, M. J. et al. One step backward is two steps forward: enhancing the hydrolysis rate of UiO-66 by decreasing $\left[\mathrm{OH}^{-}\right]$. ACS Catal. 5, 4637-4642 (2015).

36. Qin, L., Wang, X., Liu, Y. \& Wei, H. 2D-metal-organic-framework-nanozyme sensor arrays for probing phosphates and their enzymatic hydrolysis. Anal. Chem. 90, 9983-9989 (2018).

37. Walther, R. et al. Ceria nanozyme and phosphate prodrugs: drug synthesis through enzyme mimicry. ACS Appl. Mater. Interfaces 13, 25685-25693 (2021).

38. Chen, X. et al. Formulation of metal-organic framework-based drug carriers by controlled coordination of methoxy PEG phosphate: boosting colloidal stability and redispersibility. J. Am. Chem. Soc. 143, 13557-13572 (2021).

39. Radzicka, A. \& Wolfenden, R. Rates of uncatalyzed peptide bond hydrolysis in neutral solution and the transition state affinities of proteases. J. Am. Chem. Soc. 118, 6105-6109 (1996).

40. Ly, H. G. T. et al. Superactivity of MOF- 808 toward peptide bond hydrolysis. J. Am. Chem. Soc. 140, 6325-6335 (2018).

41. de Koning, M. C., van Grol, M. \& Breijaert, T. Degradation of Paraoxon and the chemical warfare agents VX, Tabun, and Soman by the metal-organic frameworks UiO-66- $\mathrm{NH}_{2}, \mathrm{MOF}-808, \mathrm{NU}-1000$, and PCN-777. Inorg. Chem. 56, 11804-11809 (2017).

42. Swaney, D. L., Wenger, C. D. \& Coon, J. J. Value of using multiple proteases for large-scale mass spectrometry-based proteomics. J. Proteome Res. 9, 1323-1329 (2010).

43. Oliyai, C. \& Borchardt, R. T. Chemical pathways of peptide degradation. IV. pathways, kinetics, and mechanism of degradation of an aspartyl residue in a model hexapeptide. Pharm. Res. 10, 95-102 (1993).

44. Flemming, H. C. et al. Biofilms: an emergent form of bacterial life. Nat. Rev. Microbiol. 14, 563-575 (2016).

45. Wiriya, Thongsomboon et al. Phosphoethanolamine cellulose: a naturally produced chemically modified cellulose. Science 359, 334-338 (2018)

46. $\mathrm{Hu}, \mathrm{Z}$. et al. Modulator effects on the water-based synthesis of $\mathrm{Zr} / \mathrm{Hf}$ metal-organic frameworks: quantitative relationship studies between modulator, synthetic condition, and performance. Cryst. Growth Des. 16, 2295-2301 (2016).

47. Park, H. J. et al. Synthesis of a Zr-based metal-organic framework with spirobifluorenetetrabenzoic acid for the effective removal of nerve agent simulants. Inorg. Chem. 56, 12098-12101 (2017).

48. Feng, D. et al. Zirconium-metalloporphyrin PCN-222: mesoporous metal-organic frameworks with ultrahigh stability as biomimetic catalysts. Angew. Chem. Int. Ed. 51, 10197-10197 (2012).

49. Feng, D. et al. A highly stable zeotype mesoporous zirconium metal-organic framework with ultralarge pores. Angew. Chem. Int. Ed. 54, 149-154 (2015).

50. Liu, W.-G. \& Truhlar, D. G. Computational linker design for highly crystalline metal-organic framework NU-1000. Chem. Mater. 29, 8073-8081 (2017).

51. Peterson, G. W. et al. Tailoring the pore size and functionality of UiO-type metal-organic frameworks for optimal nerve agent destruction. Inorg. Chem. 54, 9684-9686 (2015).

52. Li, A. et al. Chemical cleavage at aspartyl residues for protein identification. Anal. Chem. 73, 5395-5402 (2001).

\section{Acknowledgements}

We thank Profs. Yunqi Li, Susumu Kitagawa, and Kenichi Otake for their insightful discussion and inputs. This work was supported by the National Key R\&D Program of China (H.W., 2019YFA0709200), National Natural Science Foundation of China (H.W., 21874067 and 21722503), PAPD Program, Fundamental Research Funds for the Central Universities (H.W., 021314380195), Sakura Science Program and Interdisciplinary Project Funded by Graduate School of Nanjing University (S.L., 2017CL12).

\section{Author contributions}

H.W. and S.L. designed the experiments and wrote the manuscript with inputs from all authors. S.L., Z.Z., Z.T., Q.L., She. Z., Q.W., and Y.Z. carried out the material synthesis, characterisation, catalytic performance, bacterial experiments and analysed the data. B.W., M.Y., L.D., Shu. Z., R.C., W.L., C.W., H.Z., and Y.D. provided material characterisation, protein analysis and chromatograph analysis.

\section{Competing interests}

The authors declare no competing interests.

\section{Additional information}

Supplementary information The online version contains supplementary material available at https://doi.org/10.1038/s41467-022-28344-2.

Correspondence and requests for materials should be addressed to Hui Wei.

Peer review information Nature Communications thanks the anonymous reviewers for their contribution to the peer review of this work.

Reprints and permission information is available at http://www.nature.com/reprints

Publisher's note Springer Nature remains neutral with regard to jurisdictional claims in published maps and institutional affiliations.

cc) (i) Open Access This article is licensed under a Creative Commons Attribution 4.0 International License, which permits use, sharing, adaptation, distribution and reproduction in any medium or format, as long as you give appropriate credit to the original author(s) and the source, provide a link to the Creative Commons license, and indicate if changes were made. The images or other third party material in this article are included in the article's Creative Commons license, unless indicated otherwise in a credit line to the material. If material is not included in the article's Creative Commons license and your intended use is not permitted by statutory regulation or exceeds the permitted use, you will need to obtain permission directly from the copyright holder. To view a copy of this license, visit http://creativecommons.org/ licenses/by/4.0/.

(C) The Author(s) 2022 